Searches for dark matter annihilation signatures in the Segue 1 satellite galaxy with the MAGIC-I telescope

This article has been downloaded from IOPscience. Please scroll down to see the full text article.

JCAP06(2011)035

(http://iopscience.iop.org/1475-7516/2011/06/035)

View the table of contents for this issue, or go to the journal homepage for more

Download details:

IP Address: 141.34.3.153

The article was downloaded on 11/01/2012 at 10:20

Please note that terms and conditions apply. 


\section{Searches for dark matter annihilation signatures in the Segue 1 satellite galaxy with the MAGIC-I telescope}

MAGIC collaboration

J. Aleksić, ${ }^{1}$ E.A. Alvarez, ${ }^{2}$ L.A. Antonelli, ${ }^{3}$ P. Antoranz, ${ }^{4}$ M. Asensio, ${ }^{2}$ M. Backes, ${ }^{5}$ J.A. Barrio, ${ }^{2}$ D. Bastieri, ${ }^{6}$ J. Becerra González, ${ }^{7,8}$ W. Bednarek, ${ }^{9}$ A. Berdyugin, ${ }^{10}$ K. Berger, ${ }^{7,8}$

E. Bernardini, ${ }^{11}$ A. Biland, ${ }^{12}$ O. Blanch, ${ }^{1}$ R.K. Bock, ${ }^{13}$ A. Boller, ${ }^{12}$ G. Bonnoli, ${ }^{3}$ D. Borla Tridon, ${ }^{13}$ I. Braun, ${ }^{12}$ T. Bretz, ${ }^{14,26}$

A. Cañellas, ${ }^{15}$ E. Carmona, ${ }^{13}$ A. Carosi, ${ }^{3}$ P. Colin, ${ }^{13}$ E. Colombo, ${ }^{7}$ J. L. Contreras, ${ }^{2}$ J. Cortina, ${ }^{1}$ L. Cossio, ${ }^{16}$ S. Covino, ${ }^{3}$ F. Dazzi, ${ }^{16,27}$ A. De Angelis, ${ }^{16}$ E. De Cea del Pozo, ${ }^{17}$ B. De Lotto, ${ }^{16}$ C. Delgado Mendez, ${ }^{7,28}$ A. Diago Ortega, ${ }^{7,8}$ M. Doert, ${ }^{5}$ A. Domínguez ${ }^{18}$ D. Dominis Prester, ${ }^{19}$ D. Dorner, ${ }^{12}$ M. Doro, ${ }^{20, \dagger}$ D. Elsaesser, ${ }^{14}$ D. Ferenc, ${ }^{19}$ M.V. Fonseca, ${ }^{2}$ L. Font, ${ }^{20}$ C. Fruck, ${ }^{13}$ R.J. García López $^{7,8}$ M. Garczarczyk, ${ }^{7}$ D. Garrido, ${ }^{20}$ G. Giavitto, ${ }^{1}$

N. Godinović, ${ }^{19}$ D. Hadasch, ${ }^{17}$ D. Häfner, ${ }^{13}$ A. Herrero, ${ }^{7,8}$

D. Hildebrand, ${ }^{12}$ D. Höhne-Mönch, ${ }^{14}$ J. Hose, ${ }^{13}$ D. Hrupec, ${ }^{19}$

B. Huber, ${ }^{12}$ T. Jogler, ${ }^{13}$ S. Klepser, ${ }^{1}$ T. Krähenbühl, ${ }^{12}$ J. Krause, ${ }^{13}$ A. La Barbera, ${ }^{3}$ D. Lelas, ${ }^{19}$ E. Leonardo, ${ }^{4}$ E. Lindfors,,${ }^{10}$

S. Lombardi, ${ }^{6, \dagger}$ M. López, ${ }^{2}$ E. Lorenz, ${ }^{12,13}$ M. Makariev, ${ }^{21}$

G. Maneva, ${ }^{21}$ N. Mankuzhiyil, ${ }^{16}$ K. Mannheim, ${ }^{14}$ L. Maraschi, ${ }^{3}$ M. Mariotti, ${ }^{6}$ M. Martínez, ${ }^{1}$ D. Mazin, ${ }^{1,13}$ M. Meucci, ${ }^{4}$ J. M. Miranda, ${ }^{4}$ R. Mirzoyan, ${ }^{13}$ H. Miyamoto, ${ }^{13}$ J. Moldón, ${ }^{15}$ A. Moralejo, ${ }^{1}$ P. Munar-Androver, ${ }^{15}$ D. Nieto ${ }^{2, \dagger}$ K. Nilsson, ${ }^{10,29}$ R. Orito, ${ }^{13}$ I. Oya, ${ }^{2}$ S. Paiano, ${ }^{6}$ D. Paneque, ${ }^{13}$ R. Paoletti, ${ }^{4}$ S. Pardo, ${ }^{2}$ J.M. Paredes, ${ }^{15}$ S. Partini, ${ }^{4}$ M. Pasanen, ${ }^{10}$ F. Pauss, ${ }^{12}$ M.A. Perez-Torres, ${ }^{1}$ M. Persic, ${ }^{16,22}$ L. Peruzzo, ${ }^{6}$ M. Pilia, ${ }^{23}$ J. Pochon, ${ }^{7}$ F. Prada, ${ }^{18}$ P.G. Prada Moroni, ${ }^{24}$ E. Prandini, ${ }^{6}$ I. Puljak, ${ }^{19}$ I. Reichardt, ${ }^{1}$ R. Reinthal, ${ }^{10}$ W. Rhode, ${ }^{5}$ M. Ribó, ${ }^{15}$

${ }^{\dagger}$ Corresponding author. 
J. Rico, ${ }^{25,1}$ S. Rügamer, ${ }^{14}$ A. Saggion, ${ }^{6}$ K. Saito, ${ }^{13}$ T. Y. Saito, ${ }^{13}$ M. Salvati, ${ }^{3}$ K. Satalecka, ${ }^{11}$ V. Scalzotto, ${ }^{6}$ V. Scapin, ${ }^{2}$ C. Schultz, ${ }^{6}$ T. Schweizer, ${ }^{13}$ M. Shayduk, ${ }^{13}$ S. N. Shore, ${ }^{24}$ A. Sillanpää, ${ }^{10}$ J. Sitarek, ${ }^{9}$ D. Sobczynska, ${ }^{9}$ F. Spanier, ${ }^{14}$ S. Spiro, ${ }^{3}$ A. Stamerra, ${ }^{4}$ B. Steinke, ${ }^{13}$ J. Storz, ${ }^{14}$ N. Strah, ${ }^{5}$ T. Surić, ${ }^{19}$ L. Takalo, ${ }^{10}$ H. Takami, ${ }^{13}$ F. Tavecchio, ${ }^{3}$ P. Temnikov, ${ }^{21}$ T. Terzić,,${ }^{19}$ D. Tescaro, ${ }^{24} \mathrm{M}$. Teshima, ${ }^{13} \mathrm{M}$. Thom, ${ }^{5}$ O. Tibolla, ${ }^{14}$ D.F. Torres, ${ }^{25,17}$ A. Treves, ${ }^{23}$ H. Vankov, ${ }^{21}$ P. Vogler, ${ }^{12}$ R. M. Wagner, ${ }^{13}$ Q. Weitzel, ${ }^{12}$ V. Zabalza, ${ }^{15}$ F. Zandanel, ${ }^{18}$ R. Zanin, ${ }^{1}$ M. Fornasa, ${ }^{18,30, \dagger}$ R. Essig, ${ }^{31}$ N. Sehgal ${ }^{32}$ and L.E. Strigari ${ }^{32}$

${ }^{1}$ IFAE, Edifici Cn., Campus UAB, E-08193 Bellaterra, Spain

${ }^{2}$ Universidad Complutense, E-28040 Madrid, Spain

${ }^{3}$ INAF National Institute for Astrophysics, I-00136 Rome, Italy

${ }^{4}$ Università di Siena and INFN Pisa, I-53100 Siena, Italy

${ }^{5}$ Technische Universität Dortmund, D-44221 Dortmund, Germany

${ }^{6}$ Università di Padova and INFN, I-35131 Padova, Italy

${ }^{7}$ Inst. de Astrofísica de Canarias, E-38200 La Laguna, Tenerife, Spain

${ }^{8}$ Depto. de Astrofísica, Universidad de La Laguna, E-38206 La Laguna, Spain

${ }^{9}$ University of Łódź, PL-90236 Lodz, Poland

${ }^{10}$ Tuorla Observatory, University of Turku, FI-21500 Piikkiö, Finland

${ }^{11}$ Deutsches Elektronen-Synchrotron (DESY), D-15738 Zeuthen, Germany

${ }^{12}$ ETH Zurich, CH-8093 Switzerland

${ }^{13}$ Max-Planck-Institut für Physik, D-80805 München, Germany

${ }^{14}$ Universität Würzburg, D-97074 Würzburg, Germany

${ }^{15}$ Universitat de Barcelona (ICC/IEEC), E-08028 Barcelona, Spain

${ }^{16}$ Università di Udine and INFN Trieste, I-33100 Udine, Italy

${ }^{17}$ Institut de Ciències de l'Espai (IEEC-CSIC), E-08193 Bellaterra, Spain

${ }^{18}$ Inst. de Astrofísica de Andalucía (CSIC), E-18080 Granada, Spain

${ }^{19}$ Croatian MAGIC Consortium, Institute R. Boskovic, University of Rijeka and University of Split, HR-10000 Zagreb, Croatia

${ }^{20}$ Universitat Autònoma de Barcelona, E-08193 Bellaterra, Spain

${ }^{21}$ Inst. for Nucl. Research and Nucl. Energy, BG-1784 Sofia, Bulgaria

${ }^{22} \mathrm{INAF} /$ Osservatorio Astronomico and INFN, I-34143 Trieste, Italy

${ }^{23}$ Università dell'Insubria, Como, I-22100 Como, Italy

${ }^{24}$ Università di Pisa and INFN Pisa, I-56126 Pisa, Italy

${ }^{25}$ ICREA, E-08010 Barcelona, Spain

${ }^{26}$ Ecole polytechnique fédérale de Lausanne (EPFL), Lausanne, Switzerland

${ }^{27}$ Supported by INFN Padova

${ }^{28}$ Centro de Investigaciones Energéticas, Medioambientales y Tecnológicas (CIEMAT),

Madrid, Spain

${ }^{29}$ Finnish Centre for Astronomy with ESO (FINCA), Turku, Finland 
${ }^{30}$ MultiDark fellow

${ }^{31}$ Stanford Linear Accelerator Center, Stanford, California, 94309, U.S.A.

${ }^{32}$ Kavli Institute for Particle Astrophysics and Cosmology, Stanford University, Stanford, California 94305, U.S.A.

E-mail: michele.doro@uab.cat,fornasam@gmail.com,slombard@pd.infn.it, nieto@gae.ucm.es

Received March 3, 2011

Revised May 19, 2011

Accepted June 15, 2011

Published June 29, 2011

Abstract. We report the results of the observation of the nearby satellite galaxy Segue 1 performed by the MAGIC-I ground-based gamma-ray telescope between November 2008 and March 2009 for a total of 43.2 hours. No significant gamma-ray emission was found above the background. Differential upper limits on the gamma-ray flux are derived assuming various power-law slopes for the possible emission spectrum. Integral upper limits are also calculated for several power-law spectra and for different energy thresholds. The values are of the order of $10^{-11} \mathrm{ph} \mathrm{cm}^{-2} \mathrm{~s}^{-1}$ above $100 \mathrm{GeV}$ and $10^{-12} \mathrm{ph} \mathrm{cm}^{-2} \mathrm{~s}^{-1}$ above $200 \mathrm{GeV}$. Segue 1 is currently considered one of the most interesting targets for indirect dark matter searches. In these terms, the upper limits have been also interpreted in the context of annihilating dark matter particles. For such purpose, we performed a grid scan over a reasonable portion of the parameter space for the minimal SuperGravity model and computed the flux upper limit for each point separately, taking fully into account the peculiar spectral features of each model. We found that in order to match the experimental upper limits with the model predictions, a minimum flux boost of $10^{3}$ is required, and that the upper limits are quite dependent on the shape of the gamma-ray energy spectrum predicted by each specific model. Finally we compared the upper limits with the predictions of some dark matter models able to explain the PAMELA rise in the positron ratio, finding that Segue 1 data are in tension with the dark matter explanation of the PAMELA spectrum in the case of a dark matter candidate annihilating into $\tau^{+} \tau^{-}$. A complete exclusion however is not possible due to the uncertainties in the Segue 1 astrophysical factor.

Keywords: dark matter experiments, dwarfs galaxies, gamma ray experiments

ArXiv ePrint: 1103.0477 


\section{Contents}

1 Introduction 1

2 Observation of Segue 1 with the MAGIC-I telescope 2

3 Upper limits for power-law spectra 3

3.1 Differential upper limits for power-law spectra 4

3.2 Integral upper limits for power-law spectra 5

4 Constraints on dark matter models $\quad 7$

$\begin{array}{lll}4.1 & \text { Impact on PAMELA preferred region } & 15\end{array}$

5 Summary and discussion

\section{Introduction}

A major open question for modern physics is the nature of dark matter (DM): strong experimental evidences suggest the presence of this elusive component in the energy budget of the Universe (see e.g. [37, 59]), without, however, being able to provide conclusive results about its nature. One of the most popular scenarios is that of weakly interacting massive particles (WIMPs), that includes a large class of non-baryonic DM candidates with a mass typically between few tens of $\mathrm{GeV}$ and few $\mathrm{TeV}$ and an annihilation cross section of the order of weak interactions [25, 100]. Natural WIMP candidates are found, e.g., in SUperSYmmetric (SUSY) extensions of the Standard Model (SM) [57, 65], and in theories with Universal Extra-Dimensions [15, 33, 87]. Recently, a number of experimental results have appeared, which may be interpreted in terms of DM [39]: in particular, the measurement of the positron fraction in cosmic-rays in the $10-100 \mathrm{GeV}$ range by the PAMELA satellite [5], and the energy spectra of electrons and positrons above $300 \mathrm{GeV}$ measured by Fermi/LAT, H.E.S.S., and ATIC $[2,7,31]$. Lately, also the search for DM with the use of underground recoil experiments has produced interesting results $[1,9,16,24]$. In this paper we will discuss the possibility of detecting signatures of DM annihilation in the very high energy band (i.e. above $100 \mathrm{GeV}$ ) of the electromagnetic spectrum using as particularly convenient target the Segue 1 dwarf Spheroidal galaxy (dSph).

Assuming the $\Lambda \mathrm{CDM}$ cosmological model, DM structures form by hierachical collapse of small overdensities and are supposed to extend in mass down to the scale of the Earth or even below [43, 53, 91]. DM structures may also host smaller satellite structures and it has been proposed that the dSphs in the Milky Way (MW) may have formed within some of these subhalos hosted in the larger MW DM halo [58, 61, 71, 93]. So far, around two dozen dSphs have been identified in the MW. They represent excellent targets for indirect DM searches due to their very large mass-to-light ratios, low baryonic content (which disfavours gamma-ray emission from conventional astrophysical sources) and their location often at high galactic latitudes, where contamination by Galactic background is subdominant [51, 84, 89, 92].

To this class belongs Segue 1, a satellite galaxy discovered by Belokurov et al. [21] in the Sloan Extension for Galactic Understanding and Exploration (SEGUE, [97]). It is located at a distance of $23 \pm 2 \mathrm{kpc}$ from the Sun (28 kpc from the Galactic Center) at 
$(\mathrm{RA}, \mathrm{Dec})=\left(10.12^{h}, 16.08^{\circ}\right)$. Despite the fact that its nature has been debated after discovery $[21,50,76,99]$, it has now been interpreted more clearly as an ultrafaint MW satellite galaxy in ref. [88] through the identification of several new member stars (66 instead of the previous 24). This interpretation has also been confirmed in ref. [68] using a Bayesian analysis of star membership probabilities. Segue 1 was highlighted as a good target for indirect DM detection in refs. [45, 67]. We will show later on in section 4 that, following our calculations, Segue 1 results to be the most DM dominated dwarf in our galaxy, known so far. The Fermi/LAT [18] has observed Segue 1 in its survey observation mode: results from the first 3 months of data have been presented in refs. [101], while results from the first 9 months of data have been presented in refs. [102, 103]. No gamma-ray signal was detected. Implications for DM models have been considered in refs. [46, 86]. Segue 1 was also observed by the X-ray telescope onboard the SWIFT satellite. Data from this short observation $(\sim 5 \mathrm{ks})$ have been analyzed [70] where no detection is reported either.

We present here the results of the observation of the Segue 1 satellite performed by the Major Atmospheric Gamma-ray Imaging Cherenkov (MAGIC) telescope. The observation took place between November 2008 and March 2009, for a total of 43.2 hours (29.4 hours in good observational conditions after quality cuts). No significant detection above the background was found for energies above $100 \mathrm{GeV}$. In this paper we describe the data analysis and determine upper limits (ULs) for the gamma-ray emission. We extensively study the dependence of the ULs on a set of parameters like the expected energy spectrum of the source or on experimental features like the energy threshold and the angular resolution. The MAGIC-I observation is then used to put constraints on some models of DM. We focus on the case of annihilating SUSY DM candidates and compute a grid scan over a reasonable range of the SUSY parameters. Point per point we use the predicted energy spectrum to compute specific ULs, showing that the dependence on the energy spectrum is even more prominent than in the case of power-law spectra. ULs are also given for DM models that fit the PAMELA and Fermi/LAT cosmic-ray spectra.

The paper is organized as follows: in section 2 we describe the observation of Segue 1 with MAGIC-I and discuss the analysis. In section 3 the calculation of the ULs on the differential and integral flux is outlined and results are reported in the case of power-law spectra. We focus on a possible DM interpretation in section 4. Conclusions are drawn in section 5 together with a brief summary.

\section{Observation of Segue 1 with the MAGIC-I telescope}

The MAGIC experiment for ground-based gamma-ray astronomy consists of a system of two telescopes operating in stereoscopic mode since fall 2009 at the Canary Island of La Palma (28.8 ${ }^{\circ}$ N, $17.8^{\circ} \mathrm{W}, 2200 \mathrm{~m}$ a.s.l.). However, the observation of Segue 1 was carried out when only the first telescope was operating. The data were taken during dark nights between November 2008 and March 2009, for a total exposure time of 43.2 hours. The source was surveyed at zenith angles between $12.7^{\circ}$ and $33.9^{\circ}$, which guarantees a low energy threshold, in false-source tracking (wobble) mode [48], in which the pointing direction alternates every 20 minutes between two positions, offset by $\pm 0.4^{\circ}$ in RA from the source.

The data analysis was performed using the standard MAGIC-I analysis and reconstruction software. After the calibration and image cleaning $[12,13]$, the hadronic background rejection is achieved through a multivariate method called Random Forest (RF) [12, 26]. The algorithm uses the image Hillas parameters [54] and timing variables to compute a 
gamma-ray/hadron discriminator called hadronness by comparison with Monte Carlo (MC) gamma-ray simulations. Hadronness ranges from 0 (for showers confidently identified as initiated by gamma-rays) to 1 (for those clearly showing the features of a hadronic cosmic-ray initiated shower). The RF method is also used to estimate the energy of the showers. The detector MC simulation was tuned to fit the actual telescope performance at the time of the observations. After standard quality cuts in trigger rate, atmospheric condition, and a check of the distribution of basic image parameters, around 30\% of the Segue 1 data were rejected, resulting in 29.4 hours of data. The same quality cuts were applied to a sample of Crab Nebula data, taken in similar experimental conditions, to cross-check the RF routines and the analysis cuts.

With respect to the standard MAGIC-I analysis, an additional cut was introduced to account for the presence of the star $\eta$-Leonis, with apparent magnitude $V=3.5, B-V=0.02$ and $(\mathrm{RA}, \mathrm{Dec})=\left(10.12^{h}, 16.76^{\circ}\right)$, located at $0.68^{\circ}$ from the position of Segue 1 . The light of the star entered the trigger region of the MAGIC-I camera and, as a consequence, produced local inhomogeneities, which are not treated automatically by the standard analysis at energies below $200 \mathrm{GeV}$. Therefore, all events below this energy and spatially coincident with an optimized region around the position of the star in the camera were discarded. With such additional star-cuts, around $20 \%$ of the events were rejected, but the local inhomogeneities caused by the star in the trigger area were efficiently washed out. To check the effect of the cuts in the effective area and eventually in the sensitivity, the same star-cuts were applied to the Crab Nebula data, and to the MC gamma-ray events for consistency. The final energy threshold was at $100 \mathrm{GeV}$ and the star-cuts had the main effect of slightly reducing the effective area at lower energies.

The number of candidates for gamma-ray events from the direction of the source was estimated using the distribution of $|\alpha|$ angles, ${ }^{1}$ which is related to the orientation of the showers. The best set of cuts for the hadronness and $|\alpha|$ parameters were optimized on the Crab Nebula data sample, separately in logarithmic energy bins (dynamic cuts), and under the assumption of a point-like emission. In section 4 we will further discuss this issue. In figure 1, the $|\alpha|$-plot above $100 \mathrm{GeV}$ is shown. For this plot, the number of excess events was computed in a fixed fiducial signal region (static cut) with $|\alpha|<14^{\circ 2}$ and is $N_{\text {exc }}(>100 \mathrm{GeV})=-279 \pm 329$, corresponding to a significance of $-0.85 \sigma$, computed using eq. (17) of Li \& Ma [64].

In figure 2, we show the significance map for the sky region around Segue 1. For this plot the source independent DISP method was used [44]. The energy threshold in this case was raised to $200 \mathrm{GeV}$ to cope with the presence of the star in the trigger region. The significance distribution is consistent with background fluctuations.

\section{Upper limits for power-law spectra}

In the previous section we presented the Segue 1 data collected by the MAGIC-I telescope, showing that, above $100 \mathrm{GeV}$, results are consistent with no signal over the background. We derive now differential and integral flux ULs for the gamma-ray emission from the source. In

\footnotetext{
${ }^{1}$ The $|\alpha|$ parameter is defined as the angle between the major axis of an image and the direction from the image center of gravity to a reference point in the field-of-view, which is the nominal source position for the ON data, and a control background point for the OFF data [54].

${ }^{2}$ The static $|\alpha|$-cut at $14^{\circ}$ is used for display purpose. In the computation of the flux upper limits, the $|\alpha|$-cuts are instead optimized more suitably in a dynamic way in each energy bin. In the first two bins, were most of the events are concentrated, the optimized $|\alpha|$-cut is $14^{\circ}$.
} 


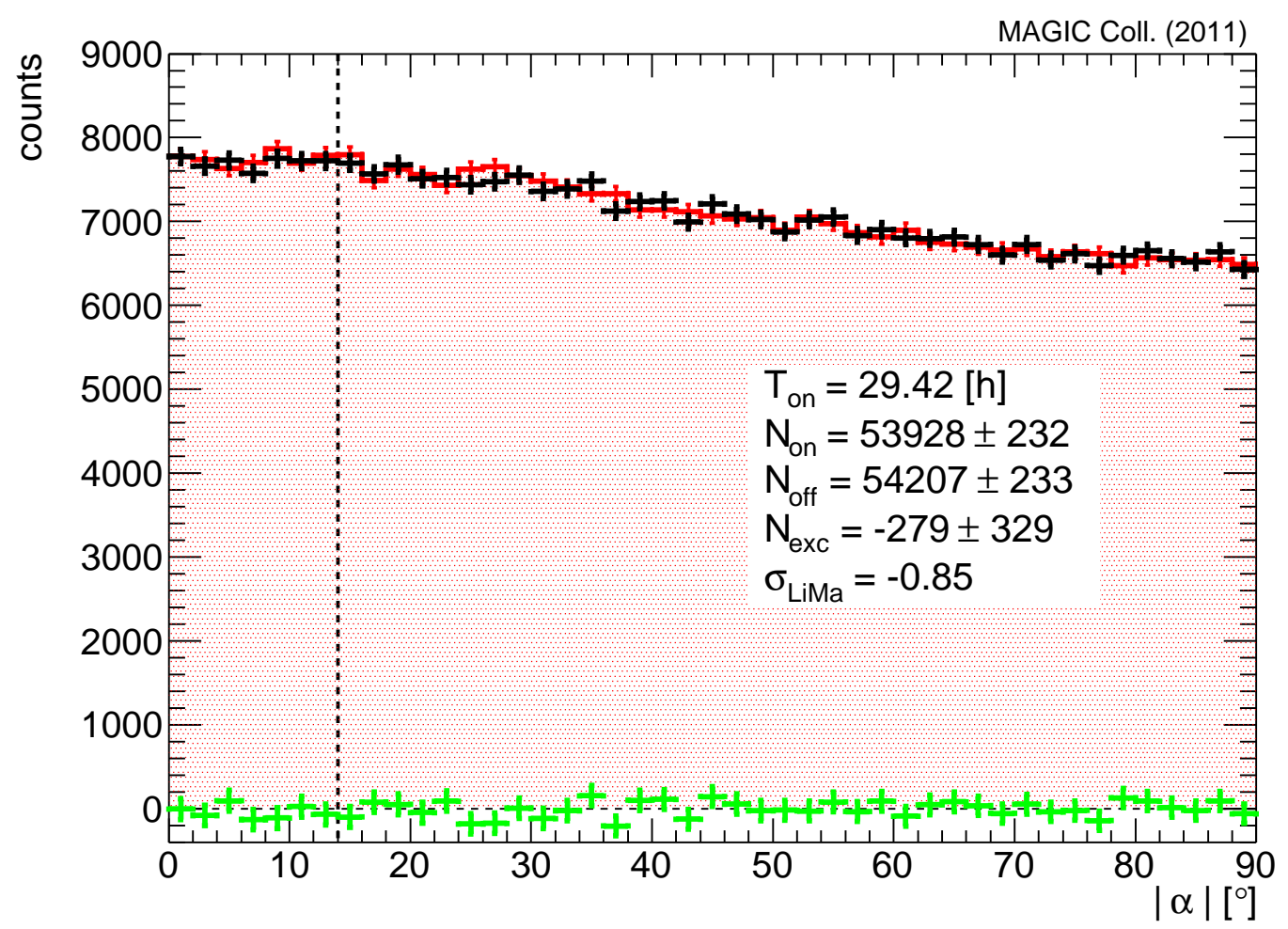

Figure 1. $|\alpha|$-plot from 29.4 hours of observation of Segue 1 above $100 \mathrm{GeV}$ with the MAGIC-I telescope. The vertical dashed line represents the fiducial region for the static cut of $|\alpha|<14^{\circ}$, where the signal is expected. Red points represent the signal (ON sample), black points the background (OFF sample), and green points their difference.

the computation of the ULs, we will distinguish between "true" energy $E$ (defined for the MC gamma-ray simulated events) and "reconstructed" energy $E_{\text {rec }}$, calculated by means of the RF method (see section 2) for the real as well as for the MC gamma-ray data.

\subsection{Differential upper limits for power-law spectra}

In order to calculate the differential flux UL in an energy bin $\Delta E_{\text {rec }}$ one can estimate the UL in the number of excess events $N_{\text {exc }}^{U L}\left(\Delta E_{\text {rec }}\right)$ following the so-called "Rolke method" [82, assuming $30 \%$ systematic uncertainty in the signal efficiency and $95 \%$ confidence level] from the $|\alpha|$-plot (obtained after the usual analysis cuts and only for reconstructed energies within the bin $\left.\Delta E_{\text {rec }}\right)$. The average effective area $A_{\text {eff }}\left(E ; \Delta E_{\text {rec }}\right)$ is calculated from the effective area for gamma-rays of true energy $E$, after all analysis cuts, including $E_{\text {rec }} \in \Delta E_{\text {rec }}$, and convolved with the energy spectrum. The latter, in this case, is assumed to be a power-law of the form $S(E) \propto\left(E / E_{*}\right)^{\Gamma}, \Gamma$ being the spectral index and $E_{*}$ the pivot energy for the particular energy bin, defined as:

$$
E_{*}=\frac{\int_{0}^{\infty} E S(E) A_{\mathrm{eff}}\left(E ; \Delta E_{\mathrm{rec}}\right) d E}{\int_{0}^{\infty} S(E) A_{\mathrm{eff}}\left(E ; \Delta E_{\mathrm{rec}}\right) d E} .
$$




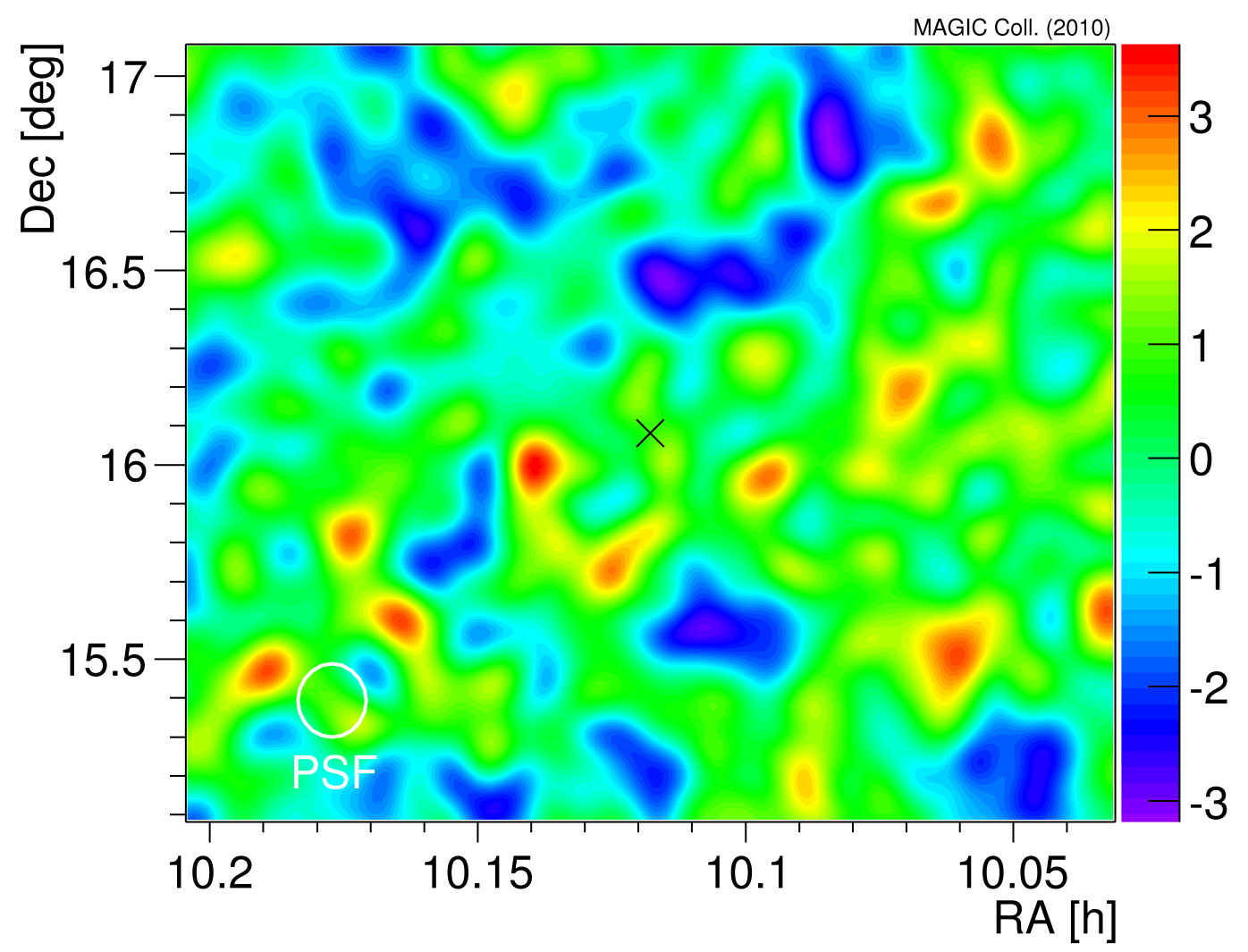

Figure 2. Significance map for events above $200 \mathrm{GeV}$ in the Segue 1 sky region. The black cross marks the position of the central core of Segue 1 and the telescope PSF is also shown. The significance distribution is consistent with background fluctuations.

Finally, the differential flux UL reads as follows:

$$
\frac{d \Phi^{U L}}{d E}\left(E_{*}\right)=\frac{N_{\mathrm{exc}}^{U L}\left(\Delta E_{\mathrm{rec}}\right)}{t_{\mathrm{eff}}} \frac{1}{\int_{0}^{\infty} A_{\mathrm{eff}}\left(E ; \Delta E_{\mathrm{rec}}\right) S(E) d E}
$$

and is computed as the UL in the number of excess events divided by the effective observation time and the average effective area weighted with the expected gamma-ray spectrum. Thus, results depend on the assumed source spectral shape (although this dependence gets less significant as the binning goes finer). The differential UL is measured in $\mathrm{ph} \mathrm{cm}^{-2} \mathrm{~s}^{-1} \mathrm{TeV}^{-1}$. table 1 summarizes the ULs in four reconstructed-energy logarithmic bins between $100 \mathrm{GeV}$ and $10 \mathrm{TeV}$ using eq. (3.2) and assuming different power-law spectra, with spectral indexes $\Gamma=-1.0,-1.5,-1.8,-2.0,-2.2,-2.4$ respectively, as done in ref. [3]. Moreover, the case of $\Gamma=-1.5$ is considered, as a reference for hadronic annihilation DM models [49]. The results are also shown in figure 3 .

\subsection{Integral upper limits for power-law spectra}

Extending the computation of the ULs to energies above a given threshold $E_{0}$, we end up with the following expression:

$$
\Phi^{U L}\left(>E_{0}\right)=\frac{N_{\mathrm{exc}}^{U L}\left(E_{\mathrm{rec}}>E_{0}\right)}{t_{\mathrm{eff}}} \frac{\int_{E_{0}}^{\infty} S(E) d E}{\int_{0}^{\infty} A_{\mathrm{eff}}\left(E ; E_{\mathrm{rec}}>E_{0}\right) S(E) d E}
$$




\begin{tabular}{|ccccccccc|}
\hline $\begin{array}{c}\Delta E \\
{[\mathrm{TeV}]}\end{array}$ & $\begin{array}{c}N_{\mathrm{ON}} / N_{\mathrm{OFF}} \\
\left(N_{\mathrm{exc}}^{U L}\right)\end{array}$ & $\begin{array}{c}\sigma_{\mathrm{Li}, \mathrm{Ma}} \\
95 \% \text { C.L. }\end{array}$ & & \multicolumn{7}{c|}{$d \Phi^{U L} / d E\left[\mathrm{TeV}^{-1} \mathrm{~cm}^{-2} \mathrm{~s}^{-1}\right]$} \\
& & & $\Gamma=-1.0$ & $\Gamma=-1.5$ & $\Gamma=-1.8$ & $\Gamma=-2.0$ & $\Gamma=-2.2$ & $\Gamma=-2.4$ \\
\hline $0.1,0.32$ & $51871 / 52271$ & -1.2 & $4.9 \cdot 10^{-11}$ & $5.2 \cdot 10^{-11}$ & $5.5 \cdot 10^{-11}$ & $5.8 \cdot 10^{-11}$ & $6.1 \cdot 10^{-11}$ & $6.5 \cdot 10^{-11}$ \\
& $(399)$ & & $(228)$ & $(211)$ & $(200)$ & $(183)$ & $(187)$ & $(180)$ \\
$0.32,1$ & $696 / 657$ & 1.1 & $3.3 \cdot 10^{-12}$ & $3.4 \cdot 10^{-12}$ & $3.6 \cdot 10^{-12}$ & $3.8 \cdot 10^{-12}$ & $4.0 \cdot 10^{-12}$ & $4.2 \cdot 10^{-12}$ \\
& $(156)$ & & $(681)$ & $(631)$ & $(603)$ & $(584)$ & $(566)$ & $(548)$ \\
$1,3.2$ & $99 / 77$ & 1.7 & $3.5 \cdot 10^{-13}$ & $3.7 \cdot 10^{-13}$ & $3.9 \cdot 10^{-13}$ & $4.0 \cdot 10^{-13}$ & $4.2 \cdot 10^{-13}$ & $4.5 \cdot 10^{-13}$ \\
& $(72)$ & & $(2060)$ & $(1917)$ & $(1835)$ & $(1782)$ & $(1730)$ & $(1681)$ \\
$3.2,10$ & $69 / 57$ & 1.1 & $5.7 \cdot 10^{-14}$ & $6.0 \cdot 10^{-14}$ & $6.4 \cdot 10^{-14}$ & $6.6 \cdot 10^{-14}$ & $7.0 \cdot 10^{-14}$ & $7.4 \cdot 10^{-14}$ \\
& $(48)$ & & $(6750)$ & $(6230)$ & $(5937)$ & $(5751)$ & $(5572)$ & $(5402)$ \\
\hline
\end{tabular}

Table 1. Differential Segue 1 flux upper limits for several power-law gamma-ray spectra in four energy bins.

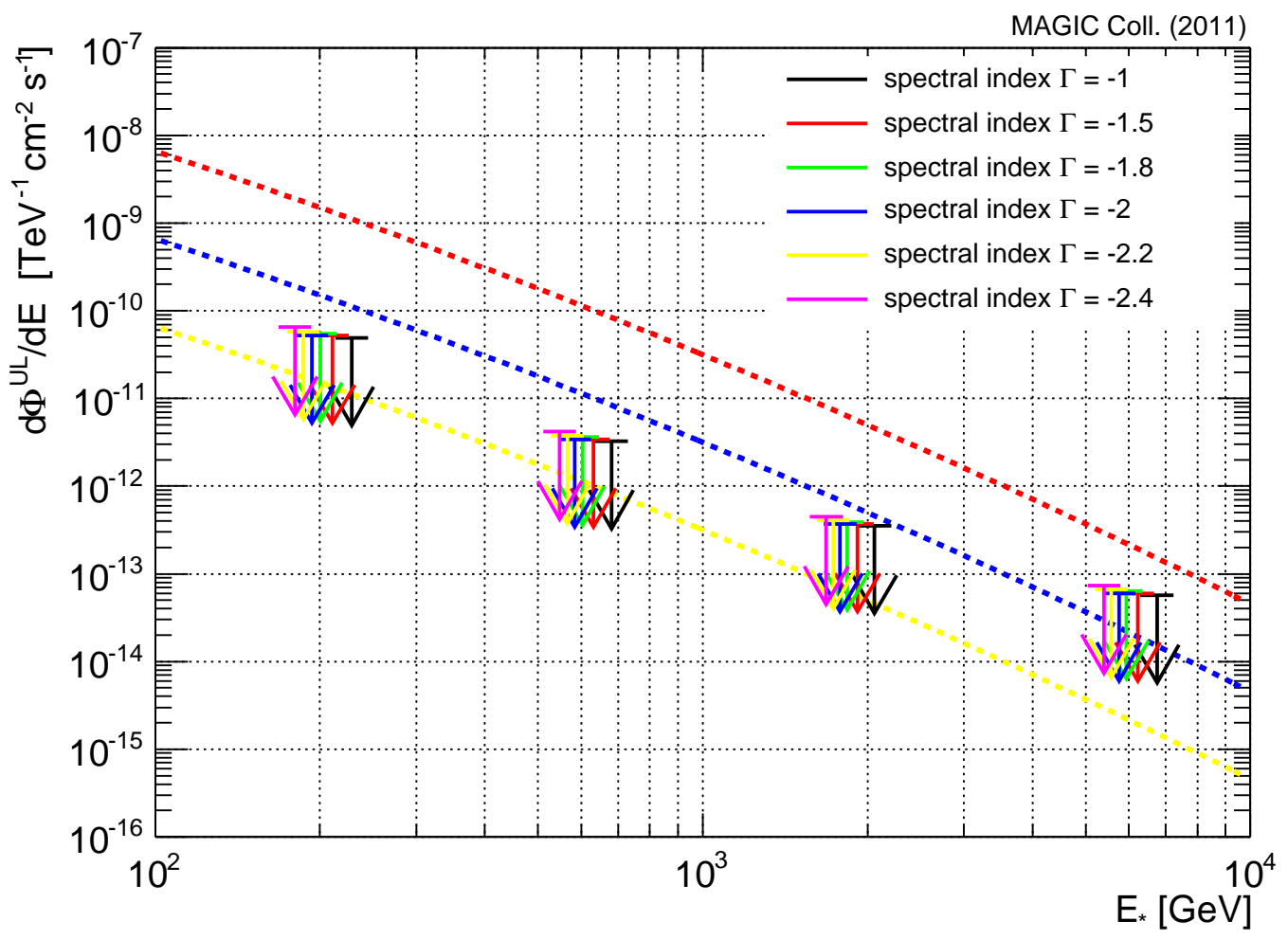

Figure 3. Differential flux upper limits from Segue 1 as in table 1 . As reference, the Crab Nebula differential flux (red dashed line) [10] and its 10\% (blue dashed line) and 1\% (yellow dashed line) fractions are also drawn.

where $S(E)$ is again the assumed energy spectrum of the source, $N_{\mathrm{exc}}^{U L}\left(E_{\mathrm{rec}}>E_{0}\right)$ is derived, as mentioned above, from the corresponding $|\alpha|$-plot for $E_{\mathrm{rec}}>E_{0}$, and the effective area is reweighted for the assumed source spectrum.

Table 2 and figure 4 present the integral ULs achieved by the MAGIC-I observation of Segue 1 for different energy thresholds and different power-law spectra with spectral index 


\begin{tabular}{|cccccccccc|}
\hline $\begin{array}{c}\mathrm{E}_{0} \\
{[\mathrm{GeV}]}\end{array}$ & $N_{\text {ON }} / N_{\text {OFF }}$ & $N_{\text {exc }}^{U L}$ & $\begin{array}{c}\sigma_{\text {Li,Ma }} \\
95 \% \text { C.L. }\end{array}$ & \multicolumn{7}{c|}{$\begin{array}{c}\Phi^{U L} / \times 10^{-12} \\
{\left[\mathrm{~cm}^{-2} \mathrm{~s}^{-1}\right]}\end{array}$} \\
& & & & $\Gamma=-1.0$ & $\Gamma=-1.5$ & $\Gamma=-1.8$ & $\Gamma=-2.0$ & $\Gamma=-2.2$ & $\Gamma=-2.4$ \\
\hline 100 & $52978 / 53301$ & 453 & -0.99 & 7.5 & 8.8 & 10.5 & 11.6 & 12.7 & 13.7 \\
126 & $18835 / 19233$ & 174 & -2.04 & 2.8 & 3.2 & 3.6 & 4.0 & 4.3 & 4.6 \\
158 & $6122 / 6374$ & 93 & -2.25 & 1.5 & 1.6 & 1.8 & 1.9 & 2.0 & 2.1 \\
200 & $3012 / 3088$ & 110 & -0.97 & 1.7 & 1.7 & 1.9 & 2.0 & 2.1 & 2.2 \\
251 & $1687 / 1654$ & 194 & 0.57 & 3.0 & 2.9 & 3.1 & 3.2 & 3.4 & 3.5 \\
316 & $1107 / 1030$ & 250 & 1.67 & 3.8 & 3.6 & 3.7 & 3.9 & 2.9 & 4.1 \\
398 & $792 / 761$ & 147 & 0.79 & 2.2 & 2.0 & 2.1 & 2.1 & 2.2 & 2.2 \\
501 & $613 / 580$ & 140 & 0.96 & 2.1 & 1.9 & 1.9 & 1.9 & 2.0 & 2.0 \\
631 & $536 / 509$ & 124 & 0.84 & 1.8 & 1.6 & 1.6 & 1.6 & 1.6 & 1.6 \\
794 & $486 / 445$ & 146 & 1.34 & 2.1 & 1.8 & 1.8 & 1.8 & 1.8 & 1.8 \\
1000 & $411 / 373$ & 135 & 1.36 & 2.0 & 1.7 & 1.6 & 1.6 & 1.6 & 1.6 \\
\hline
\end{tabular}

Table 2. Integral Segue 1 flux upper limits for several power-law spectra and different energy thresholds $E_{0}$.

$\Gamma=-1.0,-1.5,-1.8,-2.0,-2.2,-2.4$ respectively, as in the previous section. The results are comparable with the results of other IACTs on observations of dSphs $[4,6,8,11,14,96]$.

In the case of integral ULs, we see that the dependence on the different power-law gamma-ray spectra is slightly stronger than in the differential ULs case, and the UL for the $\Gamma=-1.0$ spectrum is at most a factor of two stronger than for the $\Gamma=-2.4$ spectrum for low energy thresholds.

In addition, we clearly see the effect of the statistical fluctuations (characterized by the significance of detection $\sigma_{\mathrm{Li}, \mathrm{Ma}}$ ) on the integral ULs value. The significance of detection depends on analysis cuts and the level of residual background: this has quite a strong impact on the UL of the number of events $N_{\text {exc }}^{U L}$, calculated with the Rolke method, and eventually on the flux UL (see tables 1 and 2). Quantitatively, a smaller significance corresponds to a lower (more stringent) UL: e.g., going from $\sigma_{\mathrm{Li}, \mathrm{Ma}}=-1$ to 1 , the UL gets worse by a factor of 3. This is an intrinsic feature of the stastical methods exploited in the analysis and it should be taken into account when comparing ULs from different analyses.

For this reason, in order to estimate the effect of the spectral slope and of the energy threshold on the value of the integral UL, without being biased by the fluctuation due to different values of the significance, we computed again the ULs assuming a null value for $\sigma_{\mathrm{Li}, \mathrm{Ma}}$ : in particular dashed lines in figure 4 are obtained with a significance equal to zero (i.e. with number of $\mathrm{ON}$ events equal to the number of $\mathrm{OFF}$ events in the signal region of the $|\alpha|$-plot) for different values of $\Gamma$ and $E_{0}$.

\section{Constraints on dark matter models}

In this section Segue 1 is treated as target for indirect DM searches. Assuming a particular form for Segue $1 \mathrm{DM}$ halo, we translate the ULs calculation described before into constraints on the DM annihilation rate.

The gamma-ray flux due to DM annihilations depends on $i$ ) the intrinsic DM density distribution in the source, $i$ i) the particle physics characteristics of the DM candidate and iii) the telescope energy resolution $\epsilon$, the field of view $\Delta \Omega$ within which the putative signal 


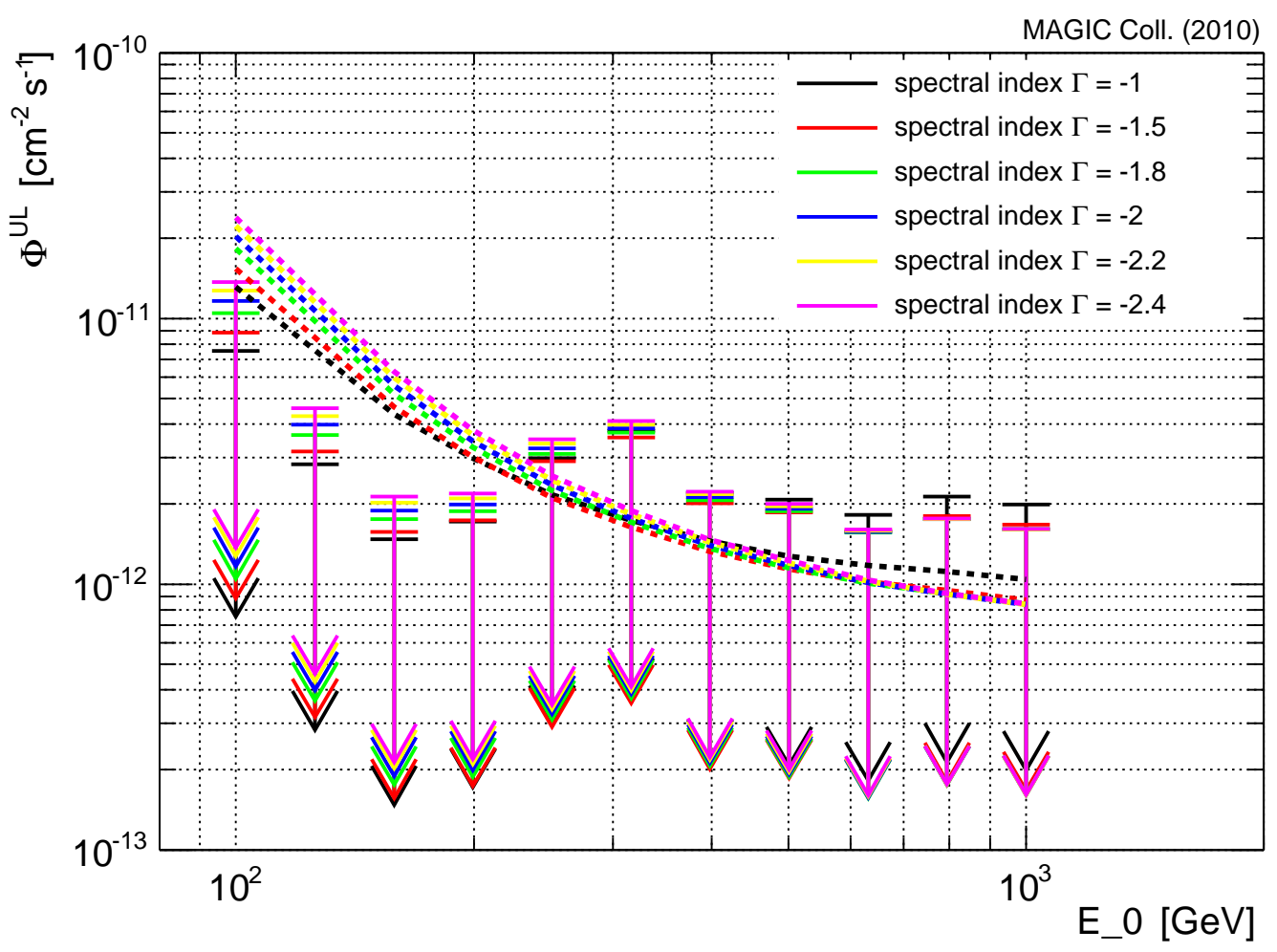

Figure 4. Integral flux upper limits from Segue 1. The arrows indicate the integral flux upper limits as in table 2 for different power-law spectra and energy thresholds. On the contrary, the dashed lines indicate the corresponding integral upper limits if zero significance $\sigma_{\mathrm{Li}, \mathrm{Ma}}$ is assumed.

is integrated, and the energy threshold $E_{0}$. It is usually factorized in two terms:

$$
\Phi\left(>E_{0}, \Delta \Omega\right)=\Phi_{\epsilon}^{P P}\left(>E_{0}\right) J(\Delta \Omega),
$$

where $\Phi_{\epsilon}^{P P}$ is the so-called particle physics factor and reads as follows:

$$
\Phi_{\epsilon}^{P P}\left(>E_{0}\right)=\frac{1}{4 \pi} \frac{\left\langle\sigma_{\mathrm{ann}} v\right\rangle}{2 m_{\chi}^{2}} \int_{E_{0}}^{m_{\chi}} \sum_{i=1}^{n} B^{i} \frac{d N_{\gamma}^{i}}{d E} d E,
$$

where $\left\langle\sigma_{\text {ann }} v\right\rangle$ is the velocity averaged annihilation cross section, $m_{\chi}$ is the DM particle mass, and $\sum_{i=1}^{n} B^{i} d N_{\gamma}^{i} / d E=d N_{\gamma} / d E$ is the sum over all the $n$ possible annihilation channels producing photons $\left(B^{i}\right.$ is the particular branching ratio for channel $i$ ).

The term $J(\Delta \Omega)$ is the astrophysical factor and is given by the line-of-sight integral over the DM density squared within a solid angle $\Delta \Omega$ :

$$
J(\Delta \Omega)=\int_{\Delta \Omega} \int_{l o s} \rho^{2}(r(s, \Omega)) d s d \Omega .
$$

Motivated by results from cosmological simulations, to describe the Segue 1 DM density distribution we used the Einasto profile [74]:

$$
\rho_{\mathrm{EIN}}(r)=\rho_{s} e^{-2 n\left[\left(r / r_{s}\right)^{1 / n}-1\right]},
$$


which produced a good fit to the subhalos simulated by the most recent $N$-body simulations [91]. eq. (4.4) is defined by three parameters: the scale density $\rho_{s}$, the scale radius $r_{s}$ and the index $n$, which typically ranges from -3 to 7 . For the computation of the astrophysical factor, we have used $\rho_{s}=1.1 \times 10^{8} \mathrm{M}_{\odot} \mathrm{kpc}^{-3}, r_{s}=0.15 \mathrm{kpc}$ and $n=3.3$, chosen among the central values after the marginalization of the likelihood used in ref. [46]. The astrophysical factor is compatible with the recent estimation of ref. [85]. The astrophysical uncertainty on $J(\Delta \Omega)$ has been estimated to be slightly larger than one order of magnitude at $2 \sigma$ level [46].

In figure 5 we show how the gamma-ray flux increases when integrating within larger regions around the center of the source. We see that the source is slightly extended compared to a representative MAGIC-I angular resolution of $0.1^{\circ}$. On the other hand, the contamination of the signal in the background region, calculated at a distance of $0.8^{\circ}$ from the "signal" region and with the same extension, is well below $1 \%$. The energy dependent $|\alpha|$ cuts applied in the analysis determine the angle of integration which fix the fraction of the total astrophysical factor that must be considered for the ULs computation. In order to get this angle we have produced a toy MC simulation for extended sources starting from point-like MC events and spreading the original gamma-ray arrival directions according to the source DM density distribution of figure 5. Since the source is only slightly extended and the assumed luminosity is very peaked at the center, this method mimics reasonably well a dedicated analysis for an extended source. We have found that the applied $|\alpha|$ cuts correspond to an angular integration of $0.14^{\circ}$ from the Segue 1 center (a solid angle of $10^{-5} \mathrm{sr}$ ). From figure 5, one can see that such angle of integration encloses $64 \%$ of the total astrophysical factor. Therefore, with the values mentioned above for scale radius and density which leads to a total astrophysical factor of $J(\Delta \Omega)=1.78 \times 10^{19} \mathrm{GeV}^{2} \mathrm{~cm}^{-5} \mathrm{sr}$, the effective astrophysical factor within the considered analysis cuts is $\widetilde{J}(\Delta \Omega)=1.14 \times 10^{19} \mathrm{GeV}^{2} \mathrm{~cm}^{-5} \mathrm{sr}$. This is, to our knowledge, the largest astrophysical factor among the known dwarf galaxies. We compared in fact our results with the astrophysical factors of table 4 of ref. [3], taking care of integrating over the same region. For a solid angle of $2.4 \times 10^{-4} \mathrm{sr}$, we get $95 \%$ of the total astrophysical factor of Segue 1, i.e. $\widetilde{J}(\Delta \Omega)=1.69 \times 10^{19} \mathrm{GeV}^{2} \mathrm{~cm}^{-5} \mathrm{sr}$, while the largest value in ref. [3] is $1.2 \times 10^{19} \mathrm{GeV}^{2} \mathrm{~cm}^{-5} \mathrm{sr}$ in the case of Draco.

Focusing now on the particle physics factor, we restrict ourselves for now to the case of a SUSY model in which the presence of a discrete symmetry ( $R$-parity) guarantees that the Lightest Supersymmetric Particle (LSP) is stable over cosmological timescales and, therefore, a good WIMP candidate. We will consider a 5-dimensional subspace of the Minimal Supersymmetric Standard Model (MSSM) called mSUGRA [30, 75], which is defined by universal masses for the gauginos $\left(m_{1 / 2}\right)$, scalars $\left(m_{0}\right)$, and trilinear couplings $\left(A_{0}\right)$, as well as by the ratio of the vacuum expectation values of the two Higgs fields $(\tan \beta)$ and the sign of the Higgsino mass term $\operatorname{sign}(\mu)$. In the majority of mSUGRA models, the LSP is the lightest neutralino $\chi$, a linear combination of the super-partners of the gauge bosons and neutral Higgs bosons.

In order to study the phenomenology of mSUGRA we performed a grid scan over the parameter space. We spanned (with linear steps) the regions indicated in table 3, chosen to provide neutralino masses within the range of detection for MAGIC-I. For each direction we considered 40 steps and in each bin along $m_{0}$ and $m_{1 / 2}$ we randomly selected a point within that bin. The scan has been done once with a positive $\operatorname{sign}(\mu)$ and then again with a negative $\operatorname{sign}(\mu)$, for a total of $5 \times 10^{6}$ points. For each mSUGRA model we used DarkSUSY 5.0.4 [52] (which includes the virtual internal Bremmstrahlung effect [27]) $i$ ) to test if the 


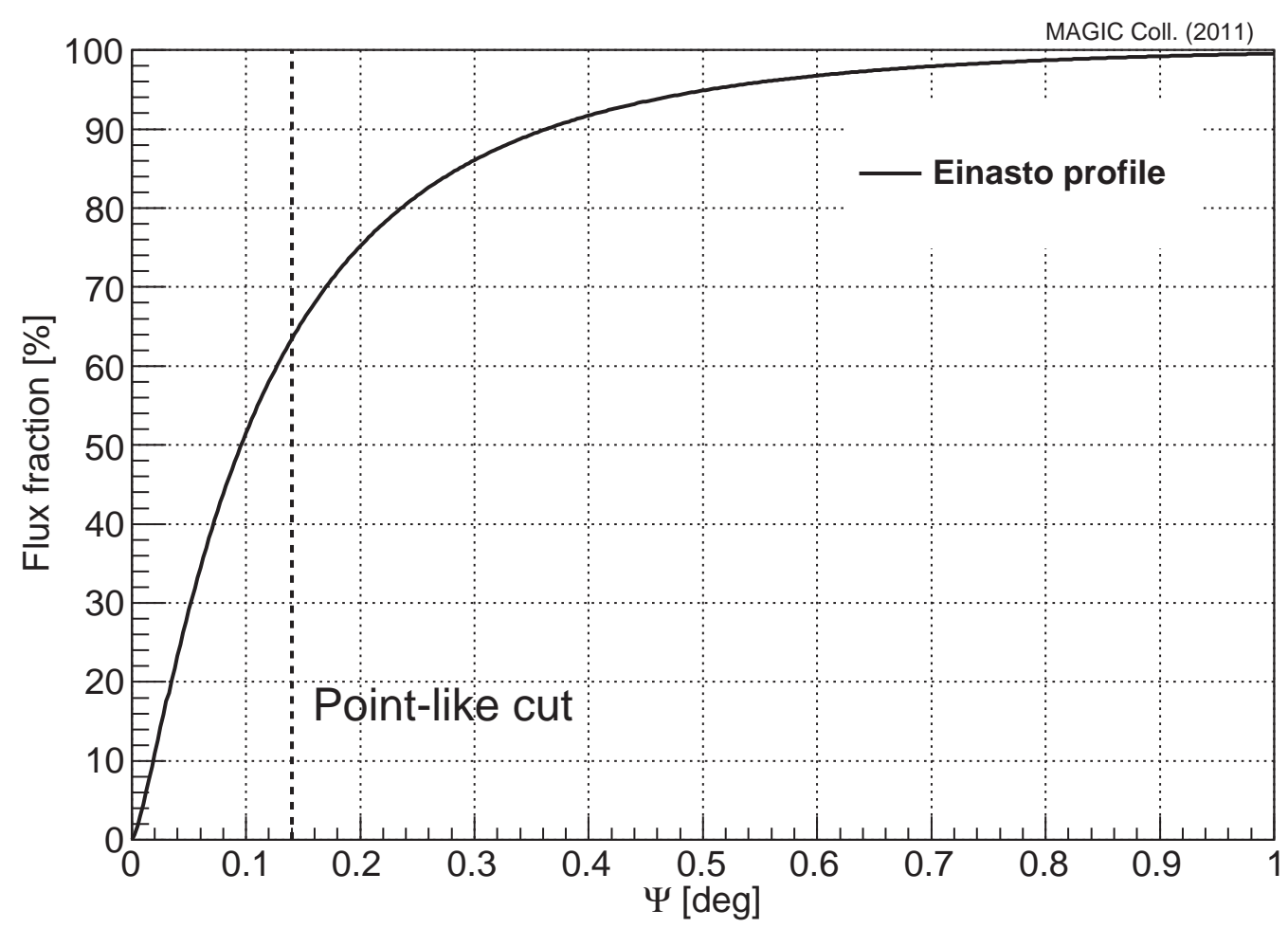

Figure 5. Fraction for squared DM density around Segue 1 integrated within an angular opening $\Psi$ with respect to the total squared DM density, as a function of $\Psi$ itself. The dashed line indicates the angular opening corresponding to the convolution of the energy dependent $|\alpha|$-cuts used in the current analysis, which were optimized for point-like sources. An Einasto profile, as defined in the text, is assumed to describe the DM density distribution.

model is physical, ii) to check if it passes the Standard Model (SM) experimental constraints implemented in the code (e.g. LEP bounds on Higgs mass $m_{h}>114 \mathrm{GeV}$, on chargino mass $m_{\chi^{+}}>103.5 \mathrm{GeV}$ and constraints from $b \rightarrow s \gamma$ ), and iii) to compute the relic density (with Isasugra $7.78[19])$.

All the models in the scan that correspond to a neutralino with a relic density compatible with the value derived by WMAP data within three times its experimental error $\sigma_{\text {WMAP }}$ [59] are plotted as crosses in figure 6 . The crosses approximatively cover the mass range between $100 \mathrm{GeV}$ and $1 \mathrm{TeV}$, due to the range that we considered for $m_{0}$ and $m_{1 / 2}$ in the scan. On the contrary, the values for the cross section span many orders of magnitude. They are mainly concentrated around a value of $10^{-26} \mathrm{~cm}^{3} \mathrm{~s}^{-1}$, but there are models that, due to particular mechanisms, are characterized by lower values for the cross section. One example is the prominent "strip" that crosses the plane from $10^{-26} \mathrm{~cm}^{3} \mathrm{~s}^{-1}$ to around $3 \times 10^{-29} \mathrm{~cm}^{3} \mathrm{~s}^{-1}$ for neutralinos that co-annihilate with stops and staus, or the "tail" at low masses (around $50 \mathrm{GeV}$ ). We stress here that the density of the points in figures 5 and 6 has no probabilistic meaning and that the region of points compatible with WMAP may extend until few times $10^{-27} \mathrm{~cm}^{3} \mathrm{~s}^{-1}$ if the range of the scan is extended (e.g. figure 25 of [20]).

For each DM model in the scan, the integral flux UL $\Phi^{U L}\left(>E_{0}\right)$ can be computed following eq. (3.3), using the Segue 1 data and the specific gamma-ray spectrum of the indi- 


\begin{tabular}{|ccc|}
\hline Parameter & Range & Steps \\
\hline$m_{0}$ & $50 ; 5000 \mathrm{GeV}$ & 40 \\
$m_{1 / 2}$ & $0 ; 5000 \mathrm{GeV}$ & 40 \\
$\tan \beta$ & $2 ; 62$ & 40 \\
$A_{0}$ & $-7000 ; 7000$ & 40 \\
$\operatorname{sign}(\mu)$ & $+;-$ & 2 \\
\hline \multicolumn{2}{|r|}{ Total number of models scanned } & $5.12 \times 10^{6}$ \\
\hline \& passing SM bounds & $2.42 \times 10^{6}$ \\
$\&$ with $\Omega_{\mathrm{DM}} h^{2}-\Omega_{\mathrm{DM}}^{\mathrm{WMAP}} h^{2}<3 \sigma_{\mathrm{WMAP}}$ & 42427 \\
$\&$ with $\left|\Omega_{\mathrm{DM}} h^{2}-\Omega_{\mathrm{DM}}^{\mathrm{WMAP}} h^{2}\right|<3 \sigma_{\mathrm{WMAP}}$ & 4180 \\
\hline \multicolumn{2}{r}{} \\
\hline
\end{tabular}

Table 3. Parameter space for the scan over mSUGRA. From Komatsu et al. [59] $\Omega_{\mathrm{DM}}^{\mathrm{WMAP}} h^{2}=0.1123$ and $\sigma_{\mathrm{WMAP}}=0.0035$.

vidual DM model. To ensure a more direct comparison with the particle physics predictions, we present our results in terms of ULs on the averaged cross section $\left\langle\sigma_{\mathrm{ann}} v\right\rangle^{U L}$, following eq. (4.2):

$$
\left\langle\sigma_{\mathrm{ann}} v\right\rangle^{U L}=\frac{8 \pi m_{\chi}^{2} \Phi^{U L}\left(>E_{0}\right)}{\widetilde{J}(\Delta \Omega) \int_{E_{0}}^{m_{\chi}} \frac{d N_{\gamma}}{d E} d E} .
$$

In section 3.2 we noticed that the integral ULs may change as a function of the energy threshold, with lower (more stringent) ULs if $E_{0}$ is larger than the experimental one (see also [28]). While this variation is more predictable in the case of power-laws, the situation may be less clear for annihilation spectra that contain features and terminate at the DM mass. An example of some gamma-ray spectra used in this analysis for a DM mass of $1 \mathrm{TeV}$ is shown in figure 7. Therefore, for each model we computed the UL for different values of energy thresholds $E_{0}$ among those listed in table 2. We start by considering the case of null significance $\sigma_{\mathrm{Li}, \mathrm{Ma}}=0$, not to be biased by fluctuations in the real $\sigma_{\mathrm{Li}, \mathrm{Ma}}$ defined above. For each annihilation channel and DM mass, we determine the optimal energy threshold $E_{\text {opt }}$ as the one with the most stringent flux UL among the set of $E_{0}$ considered.

The effect of the energy threshold optimization can be seen in figure 6 . In the bottom part of the plot, we show the models of the scan compatible with WMAP bounds as black crosses. In addition, two representative subsets are also shown using a different color coding according to their main annihilation channel (red points for branching ratio $B(b \bar{b})>0.85$, and blue points for $\left.B\left(\tau^{+} \tau^{-}\right)>0.7\right)$, which are also representatives of a soft and hard gammaray spectrum respectively (see figure 7 ). On the top part of the plot, different exclusion lines, obtained from the integral ULs, are shown. The blue dashed line is the exclusion curve obtained with the integral ULs for the $\tau^{+} \tau^{-}$annihilation channel, for $\sigma_{\mathrm{Li}, \mathrm{Ma}}=0$ and with $E_{0}=100 \mathrm{GeV}$. The blue dotted line represents the same curve but now recalculated with the optimized energy threshold method described above. One can clearly see that the optimization works at moderate and high DM masses, because at low DM masses, by increasing the energy threshold one also loses too many photons. We recall that these two curves do not represent real data, because are calculated with $\sigma_{\mathrm{Li}, \mathrm{Ma}}=0$. The thick blue solid line is finally the exclusion curve calculated with the real significance $\sigma_{\mathrm{Li}, \mathrm{Ma}}$ and the optimized energy threshold. One can now see that below roughly $1.2 \mathrm{TeV}$ the curve is more constraining than the thin blue solid one because in this regime, the best energy threshold is 


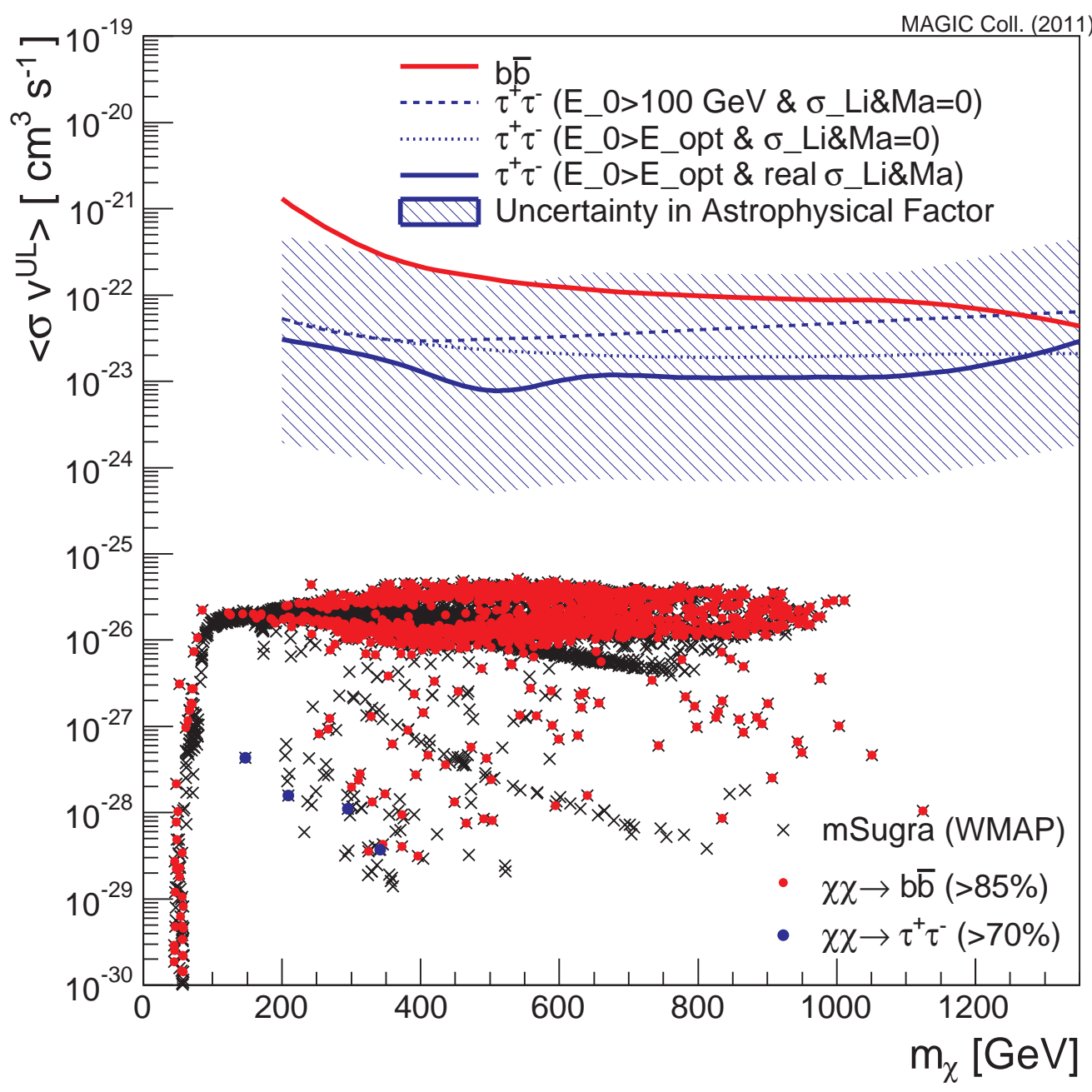

Figure 6. Annihilation cross section ULs from Segue 1 MAGIC data considering neutralino annihilating entirely into $b \bar{b}$ or into $\tau^{+} \tau^{-}$. mSUGRA models with a relic density within $3 \sigma_{\mathrm{WmAP}}$ from the WMAP value are plotted (black crosses). Among these, neutralinos annihilating mainly in $b \bar{b}$ and $\tau^{+} \tau^{-}$are indicated with red points and blue points respectively. The solid red line indicates ULs for a neutralino annihilating entirely into $b \bar{b}$ while the blue lines the case of annihilations into $\tau^{+} \tau^{-}$. The dashed blue line is calculated for a $E_{0}=100 \mathrm{GeV}$ and by imposing a null significance. The dotted blue line is also calculated from a null significance but above the optimized energy threshold $E_{\text {opt }}$ as explained the text. Finally, the thick solid blue line represents the UL calculated with the real significance and above $E_{\text {opt }}$. The $E_{\text {opt }}$ is optimized for all the DM masses. Finally, for annihilations into $\tau^{+} \tau^{-}$, the blue band covers the $2 \sigma$ uncertainty on $J_{\Theta}(\Delta \Omega)$.

between 100 and $200 \mathrm{GeV}$, where the significance is negative (from table 2). Above 1.2 TeV, the optimized energy threshold is $251 \mathrm{GeV}$ corresponding to a positive value of $\sigma_{\mathrm{Li}, \mathrm{Ma}}$, and therefore the two mentioned lines cross. Finally, the red solid line indicates the UL (with optimized energy threshold) in the case of annihilations only into $b \bar{b}$. In this case, due to the soft spectrum of this channel, the optimized energy threshold is almost all the time $100 \mathrm{GeV}$, apart from the very last points. This is reasonable, because soft spectra do not gain more 


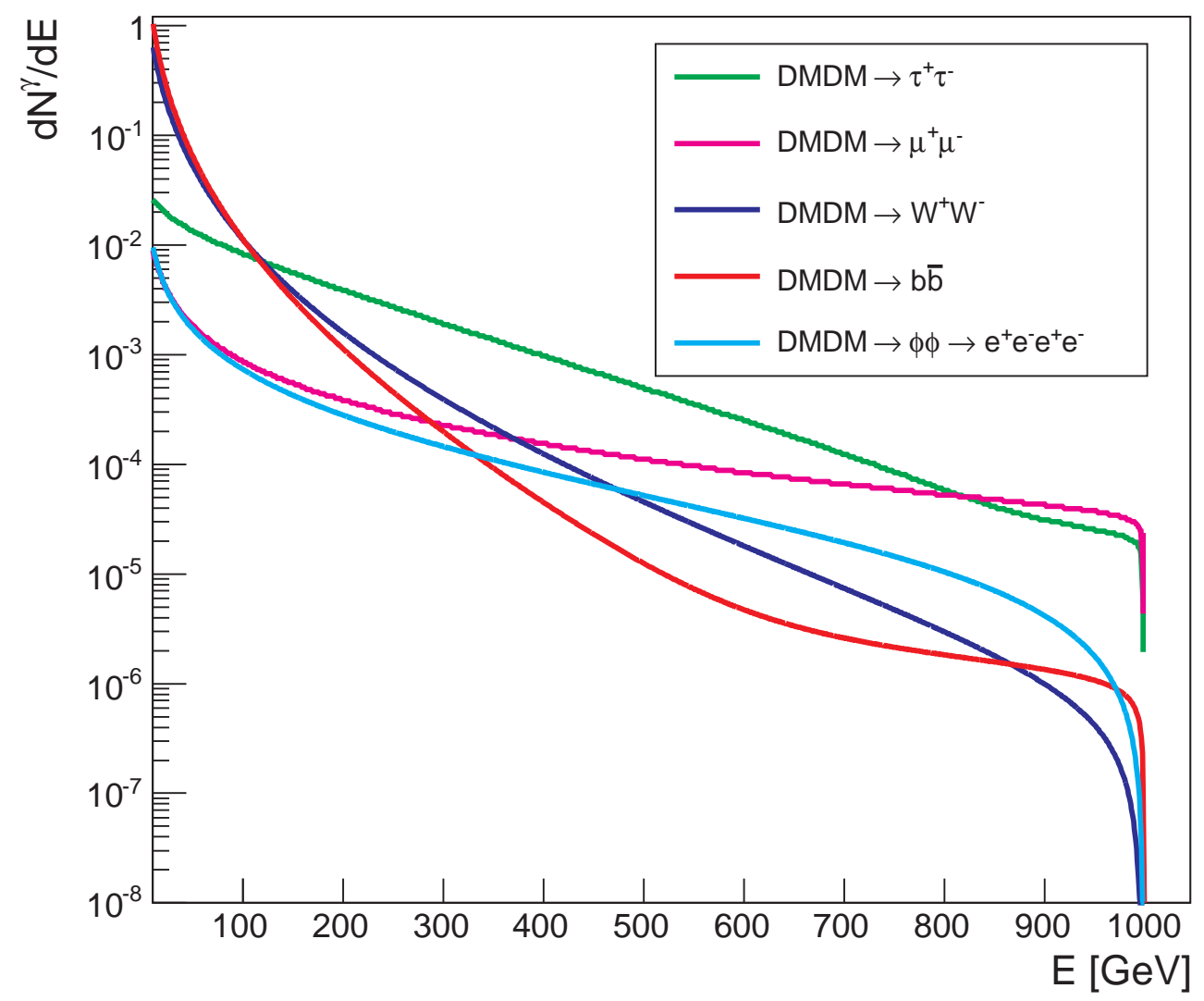

Figure 7. Spectra of different annihilation channels. For the channels $b \bar{b}, W^{+} W^{-}, \tau^{+} \tau^{-}, \mu^{+} \mu^{-}$we used the fits from ref. [29]. For $\phi \phi \rightarrow 4 e$ we used the parameterization in ref. [45] with $m_{\phi}=1 \mathrm{GeV}$.

by selecting higher energy photons. In the same figure, it is also possible to see how the ULs depend on the shape of the energy spectrum: differences can be larger than one order of magnitude (see also [3]) and, as expected, hard spectra are more constraining. Finally, in the case of annihilations into $\tau^{+} \tau^{-}$, we also indicate (by means of the blue band) the uncertainty in the astrophysical factor at the $2 \sigma$ level, showing how strongly it affects our ULs and prospects for detection.

In figure 8 we plot again the $\left\langle\sigma_{\mathrm{ann}} v\right\rangle$ predictions (same as in figure 6) for the points in the scan (full circles), together with the corresponding ULs calculated above $E_{\text {opt }}$ with the method described above (full squares). In addition, all the models in the scan that correspond to a neutralino with a relic density lower than the value derived by WMAP data plus three times its experimental error $\sigma_{\mathrm{WMAP}}$ [59] are also plotted as grey crosses. In this case, since the standard freeze-out predicts a too small relic density, we would require a non-thermal production mechanism for the DM to recover the correct relic density. We are not considering the case of multi-component DM: if the neutralino is responsible for only a fraction of the total DM density, it would be reasonable to rescale the value of the Segue 1 astrophysical factor (and consequenty the UL) by the same fraction. It can be seen that, for large neutralino masses, ULs concentrate around $10^{-22} \mathrm{~cm}^{3} \mathrm{~s}^{-1}$, while at lower masses, the distribution is wider, because models with a low gamma-ray flux above the threshold are 


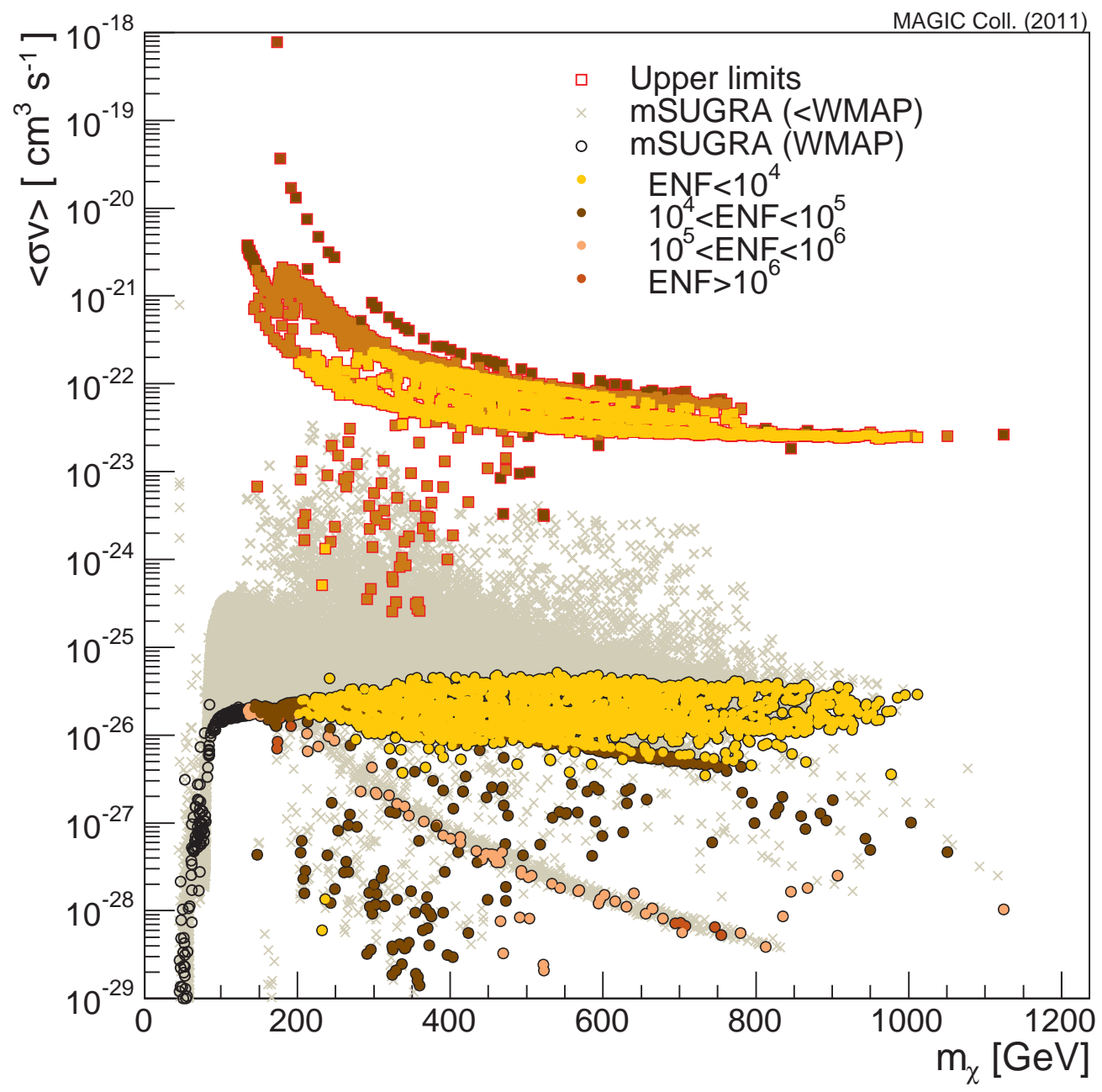

Figure 8. Annihilation cross section ULs from Segue 1 MAGIC data computed for individual points in the scan. Grey crosses indicate the annihilation cross section value for those points in the scan (see table 3) that pass the SM constraints and with a relic density lower than WMAP bound. The full circles only consider models within $3 \sigma_{\mathrm{WMAP}}$ from WMAP. For each of these full circles the UL on the cross section can be computed from the Segue 1 data (after energy threshold optimization) and it is indicated here by a square. Circles and squares are color coded in terms of the enhancement factor (see eq. (4.6)).

able to produce only loose ULs (as in the case of squares at low masses with UL of the order of $10^{-20}-10^{-19} \mathrm{~cm}^{3} \mathrm{~s}^{-1}$ ). We stress that each point in the scan should be compared to its own UL, and hence the apparent overlap in figure 8 between the ULs (plotted as squares) and some models with a relic density below the WMAP value (grey crosses) does not imply that any of these models is excluded. In order to avoid this possible misunderstanding we decide to compute point per point what we call enhancement factors (ENFs) defined as the ratio between the UL on the averaged cross section and the value predicted by mSUGRA:

$$
\mathrm{ENF}=\left\langle\sigma_{\mathrm{ann}} v\right\rangle^{U L} /\left\langle\sigma_{\mathrm{ann}} v\right\rangle
$$


This indicates how much the cross section of the particular model should be increased in order to make it detectable. In these terms, one can easily understand which points can be excluded by MAGIC-I data on Segue 1 since they would be associated to an ENF smaller than one. In figure 8 the color coding is chosen in terms of the ENFs: yellow for points with an ENF smaller than $10^{4}$, orange for models with $10^{4}<\mathrm{ENF}<10^{5}$, red if $10^{5}<\mathrm{ENF}<10^{6}$ and brown if $\mathrm{ENF}>10^{6}$.

In figure 9 the ENFs are also plotted as a function of the mass for models compatible with WMAP value of the relic density (red crosses) and below (black crosses). The panel in the upper right of the figure indicates the ENF distribution for the two sets of models. For those compatible with WMAP (red crosses), the lowest ENF is of the order of $10^{3}$. Figure 8 tells us that the models charactized by the lowest ENF are those with the largest annihilation cross section and a neutralino mass above $200 \mathrm{GeV}$. On the contrary, the majority of the points in the scan have an ENF $>10^{4}$. Moreover, it can also be seen that the distribution of ENFs is quite wide. As commented before, this large spread is due to the very high (less constraining) ULs relative to models with small neutralino masses, as already pointed out in refs. [11, 14], and in general to models with a low gamma-ray flux above the energy threshold. In the case of models with a relic density below the WMAP value (black crosses), the situation is slightly better: their intrinsic higher cross sections make them closer to their ULs, the ENF peaks at values somewhat lower than those for points compatible with WMAP and the distribution extends to lower values. We have around 160 points with $\mathrm{ENF}<20,40$ with $\mathrm{ENF}<10$ and 5 with $\mathrm{ENF}<5$ even if the exact numbers depend on the number of points scanned.

For the sake of completeness, we point out that the contribution of monochromatic lines to the annihilation spectrum is neglected in this study, due to the fact that their contribution is subdominant with respect to the continuum emission [22].

\subsection{Impact on PAMELA preferred region}

Despite the fact that several astrophysical explanations have been proposed to describe the rise in the energy spectrum of the positron fraction $e^{+} /\left(e^{+}+e^{-}\right)$measured by PAMELA $[5,55,81,98]$, an interpretation in terms of DM is still possible (see, e.g. [17, 23, 34-36, 47, 69, 80]. Typically, the DM particle has to be heavy and annihilate mainly to leptons. One possibility that has been largely studied is that the annihilation to leptons occurs through the production of an intermediate state $\phi$, mediator of a new, long range, attractive force [17]. However, a very large annihilation cross section is required, about a factor $100-1000$ larger than the canonical value derived for thermal production of $\left\langle\sigma_{\text {ann }} v\right\rangle \sim 10^{-26} \mathrm{~cm}^{3} \mathrm{~s}^{-1}$. In the following, we test our ULs against some of the models proposed in the literature that fit the PAMELA data. The regions in the $\left(m_{\chi},\left\langle\sigma_{\text {ann }} v\right\rangle\right)$ plane that provide a good fit to the PAMELA data are shown in figure 10 for a DM candidate annihilating into $\mu^{+} \mu^{-}, \tau^{+} \tau^{-}$and for the case of the intermediate state $\phi$ decaying to $e^{+} e^{-}$, with $m_{\phi}=1 \mathrm{GeV}$. These regions have been adapted from ref. [69] after rescaling from a local DM density of $0.3 \mathrm{GeV} / \mathrm{cm}^{3}$ to $0.43 \mathrm{GeV} / \mathrm{cm}^{3}$ [83].

Using again the specific DM annihilation spectra, we plot in figure 10 the ULs obtained from the Segue 1 data. We can see that, in this case, the ENFs needed to meet the PAMELAfavoured region are much smaller than for mSUGRA, and in the case of annihilation into $\tau^{+} \tau^{-}$our ULs are probing the relevant regions. However, we recall that the uncertainty in the astrophysical factor (see figure 6) is large: if future data on Segue 1 point to an astrophysical factor close to the upper end of the currently allowed range, our observations of Segue 1 might be able to confirm the exclusion of the PAMELA region for DM particles 


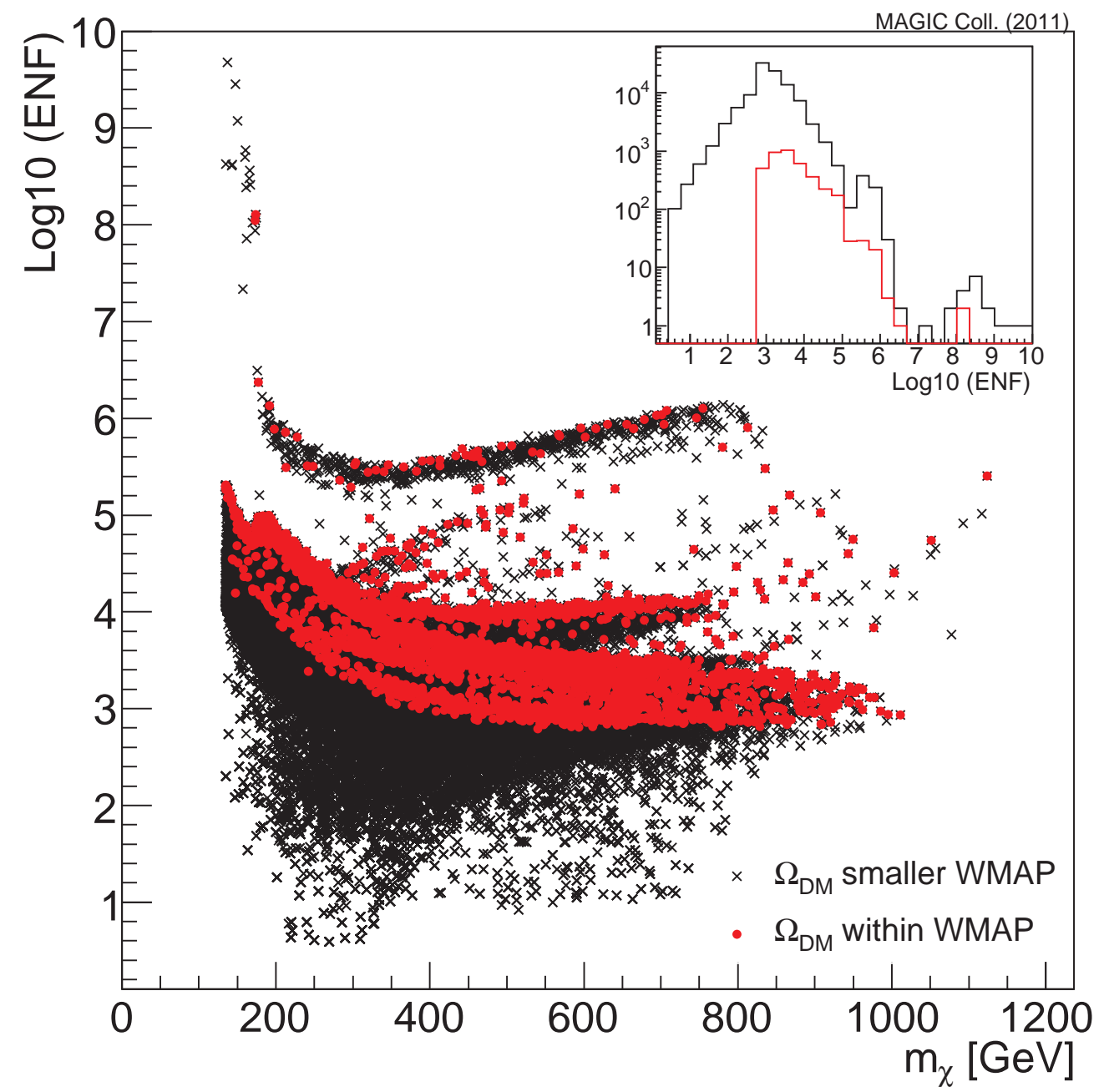

Figure 9. Enhancement factors as a function of the DM mass for models in the scan providing a relic density compatible with WMAP value (red crosses) or below (black crosses). The panel in the upper right part indicates the distribution of the ENFs for these two sets of models with the same color coding.

annihilating in $\tau^{+} \tau^{-}$, at least for massive DM candidates. Note that for the annihilation into $\phi \phi \rightarrow 4 e$, an additional Sommerfeld enhancement due to the lower DM velocity dispersion in Segue 1 compared to the DM velocity dispersion in the MW halo may effectively shift up the PAMELA preferred region by an order of magnitude, at least for low enough $\phi$ masses (see also ref. [46]).

\section{Summary and discussion}

In the present paper we presented the observation of the ultra-faint satellite galaxy Segue 1 performed by the MAGIC-I telescope (single telescope mode) over 29.4 hours of selected data. A description of the data analysis is reported with the result of no detection above the background for energies larger than $100 \mathrm{GeV}$ (sections 2). 


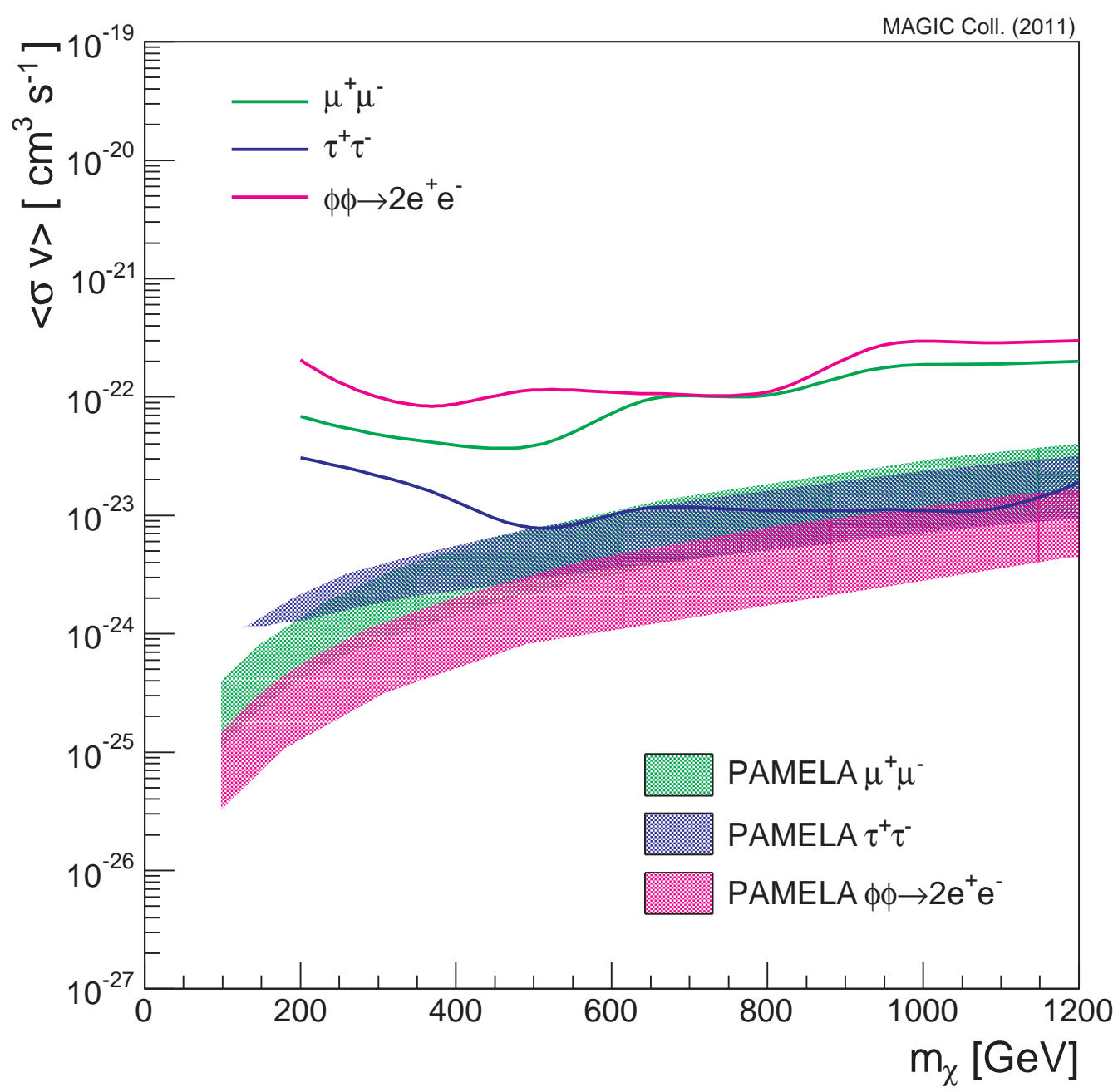

Figure 10. Exclusion lines for a neutralino DM annihilating exclusively into $\mu^{+} \mu^{-}$(green lines) or $\tau^{+} \tau^{-}$(blue line) and for a DM candidate interacting with a light intermediate state $\phi$ decaying into a pair of electrons (pink line). The same annihilation channels (with the same color coding) are considered to draw the regions in the plane that provide a good fit to the PAMELA measurement of the energy spectrum of the positron fraction. The regions are taken from ref. [46], which are adapted from ref. [69]. We used an astrophysical factor of $\widetilde{J}(\Delta \Omega)=1.14 \times 10^{19} \mathrm{GeV}^{2} \mathrm{~cm}^{-5} \mathrm{sr}$.

The result is used to compute ULs on the gamma-ray emission from the source, assuming different power-law energy spectra. The computation is done first in energy bins (table 1 and figure 3 ) and then for integral ULs above different energy thresholds (table 2 and figure 4). In all cases, we averaged the effective area reweighting it with the specific gamma-ray spectrum, which allowed us to determine how much the ULs depend on the specific spectrum. We also pointed out the fact that one can get more stringent ULs if computed above energies larger than the experimental energy threshold, as a result of the interplay between the larger sensitivity of the experiment at moderate energies and the assumed spectrum.

We focused then on indirect detection of DM and produced ULs on the annihilation cross section for a large scan of neutralino models within the mSUGRA scenario (figure 8). The 
ULs are derived separately for each point in the scan in order to completely account for the dependence on the specific spectral shape. Results indicate that ULs are quite dependent on the energy spectrum and a general exclusion plot cannot be drawn to constrain the parameter space (figure 8). For this reason, we find it quite useful to provide the results in terms of enhancement factors, defined as the intrinsic flux boost needed to meet detection (eq. (4.6)). Results are shown in figure 9. A mininum boost is found of the order of $10^{3}$ (for models compatible with WMAP) while "typical" values are at $10^{4-5}$. However, if we loosen the constraint and request only that the SUSY models do not overshoot the WMAP value for the relic density, then the situation improves since we can have ENFs as low as a few. In these terms MAGIC-I data on Segue 1 are not so far from excluding portions of mSUGRA.

We have also discussed how MAGIC-I data on Segue 1 can be used to test the PAMELA results on cosmic-rays. Current results are probing the PAMELA preferred region for the case that DM annihilates into $\tau^{+} \tau^{-}$. Future improved measurements of the kinematics of the stars in Segue 1 may decrease the uncertainty in the line-of-sight integral over the DM density squared and rule out this region.

The robustness of our results depends mainly on the assumptions on the astrophysical factor, since an uncertainty of two orders of magnitude (at $2 \sigma$ ) remains, as estimated in ref. [46]. Our result can be considered conservative since they do not take into account intrinsic contributions to the flux from the presence of substructures in the "smooth" DM halo profile of Segue 1 (which is already "per se" a substructure of the larger MW halo). The effects of substructures most likely increase the flux by a factor of a few at the most $[43,67,85,91]$. A second contribution to the flux, unaccounted for in our calculation, comes from a particle physics mechanism known as the Sommerfeld effect, which may additionally boost up the predictions for the gamma-ray emission from DM annihilation. Its effect on the predicted fluxes from halo substructures have been studied in refs. [46, 62]. The most spectacular effects are present for some resonant values of the DM mass where the flux can be increased by a factor of $\sim 10^{4}$. How these models can be constrained by dSphs observation has been studied in refs. [46, 79]. Moreover, secondary gamma-ray emission can be expected, that may enhance the predicted flux from Segue 1 and make our ULs more constraining. As studied in ref. [38], the main mechanism for secondary emission is Inverse Compton of electrons and positrons produced in DM annihilations with CMB and starlight photons. The contribution of the Inverse Compton with the CMB has been computed in the case of dSphs in ref. [3] where it can be seen that the UL on the annihilation cross section can improve of one order of magnitude for a DM mass around $1 \mathrm{TeV}$.

It is beyond the goal of this paper to discuss constraints on other DM scenarios. As a brief comment, we simply recall that models with Universal Extra Dimensions predict a stable particle whose annihilation signatures are typically harder than the ones in mSUGRA. For these models, one can use the ULs we presented for the $\tau^{+} \tau^{-}$channel, which can be taken as reference for hard spectra, and therefore draw conclusions similar to ours. Furthermore, for the case of decaying DM (that can also fit the PAMELA and Fermi/LAT-HESS results), one can rescale our results accounting for a different astrophysical factor (depending on the DM density and not on the DM density squared) and constrain the decay timescale instead of the annihilation cross section.

Comparing MAGIC-I results on Segue 1 with those of Fermi/LAT, the different energy range covered by the two experiments implies that the latter is more constraining for low mass DM candidates, while MAGIC (or Cherenkov telescopes in general) can produce better ULs only for DM heavier than few hundreds of GeV. For low mass neutralinos, it is already 
possible for Fermi/LAT to exclude some of the mSUGRA models with a relic density smaller than what was measured by WMAP [3]. With 5 years of data, Fermi/LAT may be able to probe some of the points of the parameter space considered here. In case of no detection, this will have the effect of excluding most of the models with large ENFs, shown in figure 9, which normally correspond to low-mass neutralinos. On the other hand, Fermi/LAT will not probe the larger DM masses shown in figure 8 and figure 9, where IACTs are more sensitive. In this direction, we recall that with the new stereoscopic system, MAGIC-stereo allows for improved background rejection specially below $100 \mathrm{GeV}$ (and therefore higher sensitivity), improved energy and angular resolutions, and a lower energy threshold [40].

In conclusion, while the observations presented here did not result in a detection, and the ULs require still quite high (and in some cases unmotivated) flux enhancement factors to actually match the experiment sensitivity, an analysis like the one presented here is able to point out details and features that can be important for future deep exposures of this or similar objects, using facilities with much improved sensitivity, e.g. the planned Cherenkov Telescope Array [56].

\section{Acknowledgments}

We would like to thank the Instituto de Astrofísica de Canarias for the excellent working conditions at the Observatorio del Roque de los Muchachos in La Palma. The support of the German BMBF and MPG, the Italian INFN, the Swiss National Fund SNF, and the Spanish MICINN is gratefully acknowledged. This work was also supported by the Marie Curie program, by the CPAN CSD2007-00042 and MultiDark CSD2009-00064 projects of the Spanish Consolider-Ingenio 2010 programme, by grant DO02-353 of the Bulgarian NSF, by grant 127740 of the Academy of Finland, by the YIP of the Helmholtz Gemeinschaft, by the DFG Cluster of Excellence "Origin and Structure of the Universe", and by the Polish MNiSzW grant 745/N-HESS-MAGIC/2010/0.

The authors wish to thank A. de la Cruz Dombriz and J.A.R. Cembranos for useful discussions and feedbacks and the anonymous referee for the careful reading and useful comments on the first version of this paper.

\section{References}

[1] CoGeNT collaboration, C.E. Aalseth et al., Results from a Search for Light-Mass Dark Matter with a P-type Point Contact Germanium Detector, Phys. Rev. Lett. 106 (2011) 131301 [arXiv: 1002 .4703] [SPIRES].

[2] The Fermi LAT collaboration, A.A. Abdo et al., Measurement of the Cosmic Ray e+ plus espectrum from $20 \mathrm{GeV}$ to $1 \mathrm{TeV}$ with the Fermi Large Area Telescope, Phys. Rev. Lett. 102 (2009) 181101 [arXiv:0905.0025] [SPIRES].

[3] A.A. Abdo et al., Observations of Milky Way Dwarf Spheroidal galaxies with the Fermi-LAT detector and constraints on Dark Matter models, Astrophys. J. 712 (2010) 147 [arXiv: 1001.4531] [SPIRES].

[4] THE VERITAS collaboration, et al., VERITAS Search for VHE Gamma-ray Emission from Dwarf Spheroidal Galaxies, Astrophys. J. 720 (2010) 1174 [arXiv:1006.5955] [SPIRES].

[5] PAMELA collaboration, O. Adriani et al., An anomalous positron abundance in cosmic rays with energies 1.5-100 GeV, Nature 458 (2009) 607 [arXiv:0810.4995] [SPIRES]. 
[6] HESS collaboration, . F. Aharonian, Observations of the Sagittarius Dwarf galaxy by the H.E.S.S. experiment and search for a Dark Matter signal, Astropart. Phys. 29 (2008) 55 [arXiv:0711.2369] [SPIRES].

[7] H.E.S.S. collaboration, F. Aharonian et al., Probing the ATIC peak in the cosmic-ray electron spectrum with H.E.S.S, Astron. Astrophys. 508 (2009) 561 [arXiv:0905.0105] [SPIRES].

[8] HESS collaboration,. F. Aharonian, A search for a dark matter annihilation signal towards the Canis Major overdensity with H.E.S.S, arXiv:0809.3894 [SPIRES].

[9] ThE CDMS-II collaboration, Z. Ahmed et al., Dark Matter Search Results from the CDMS II Experiment, Science 327 (2010) 1619 [arXiv:0912.3592] [SPIRES].

[10] MAGIC collaboration, J. Albert et al., VHE Gamma-Ray Observation of the Crab Nebula and its Pulsar with the MAGIC telescope, Astrophys. J. 674 (2008) 1037 [arXiv:0705.3244] [SPIRES].

[11] MAGIC collaboration, J. Albert et al., Upper limit for gamma-ray emission above 140 GeV from the dwarf spheroidal galaxy Draco, Astrophys. J. 679 (2008) 428 [arXiv:0711.2574] [SPIRES].

[12] J. Albert et al., Implementation of the Random Forest Method for the Imaging Atmospheric Cherenkov Telescope MAGIC, Nucl. Instrum. Meth. A 588 (2008) 424 [arXiv:0709.3719] [SPIRES].

[13] MAGIC collaboration, J. Albert et al., FADC signal reconstruction for the MAGIC telescope, Nucl. Instrum. Meth. A 594 (2008) 407 [astro-ph/0612385] [SPIRES].

[14] MAGIC collaboration, E. Aliu et al., MAGIC upper limits on the VHE gamma-ray emission from the satellite galaxy Willman 1, Astrophys. J. 697 (2009) 1299 [arXiv:0810.3561] [SPIRES].

[15] T. Appelquist, H.-C. Cheng and B.A. Dobrescu, Bounds on universal extra dimensions, Phys. Rev. D 64 (2001) 035002 [hep-ph/0012100] [SPIRES].

[16] XENON100 collaboration, E. Aprile et al., First Dark Matter Results from the XENON100 Experiment, Phys. Rev. Lett. 105 (2010) 131302 [arXiv:1005.0380] [SPIRES].

[17] N. Arkani-Hamed, D.P. Finkbeiner, T.R. Slatyer and N. Weiner, A Theory of Dark Matter, Phys. Rev. D 79 (2009) 015014 [arXiv:0810.0713] [SPIRES].

[18] LAT collaboration, W.B. Atwood et al., The Large Area Telescope on the Fermi Gamma-ray Space Telescope Mission, Astrophys. J. 697 (2009) 1071 [arXiv:0902.1089] [SPIRES].

[19] F.E. Paige, S.D. Protopopescu, H. Baer and X. Tata, ISAJET 7.69: A Monte Carlo event generator for $p p, \bar{p} p$ and $e^{+} e^{-}$reactions, hep-ph/0312045 [SPIRES].

[20] E.A. Baltz et al., Pre-launch estimates for GLAST sensitivity to Dark Matter annihilation signals, JCAP 07 (2008) 013 [arXiv:0806.2911] [SPIRES].

[21] SDSS collaboration, V. Belokurov et al., Cats and Dogs, Hair and A Hero: A Quintet of New Milky Way Companions, Astrophys. J. 654 (2007) 897 [astro-ph/0608448] [SPIRES].

[22] L. Bergstrom, P. Ullio and J.H. Buckley, Observability of gamma rays from dark matter neutralino annihilations in the Milky Way halo, Astropart. Phys. 9 (1998) 137 [astro-ph/9712318] [SPIRES].

[23] L. Bergstrom, J. Edsjo and G. Zaharijas, Dark matter interpretation of recent electron and positron data, Phys. Rev. Lett. 103 (2009) 031103 [arXiv:0905.0333] [SPIRES].

[24] DAMA collaboration, R. Bernabei et al., First results from DAMA/LIBRA and the combined results with DAMA/NaI, Eur. Phys. J. C 56 (2008) 333 [arXiv:0804.2741] [SPIRES].

[25] G. Bertone, D. Hooper and J. Silk, Particle dark matter: Evidence, candidates and constraints, Phys. Rept. 405 (2005) 279 [hep-ph/0404175] [SPIRES]. 
[26] L. Beiman, Random Forests, Mach. Learn. 45 (2001) 5.

[27] T. Bringmann, L. Bergstrom and J. Edsjo, New Gamma-Ray Contributions to Supersymmetric Dark Matter Annihilation, JHEP 01 (2008) 049 [arXiv:0710.3169] [SPIRES].

[28] T. Bringmann, M. Doro and M. Fornasa, Dark Matter signals from Draco and Willman 1: Prospects for MAGIC II and CTA, JCAP 01 (2009) 016 [arXiv:0809.2269] [SPIRES].

[29] J.A.R. Cembranos, A. de la Cruz-Dombriz, A. Dobado, R.A. Lineros and A.L. Maroto, Photon spectra from WIMP annihilation, Phys. Rev. D 83 (2011) 083507 [arXiv: 1009.4936] [SPIRES].

[30] A.H. Chamseddine, R.L. Arnowitt and P. Nath, Locally Supersymmetric Grand Unification, Phys. Rev. Lett. 49 (1982) 970.

[31] J. Chang et al., An excess of cosmic ray electrons at energies of 300-800 GeV, Nature 456 (2008) 362.

[32] S. Chang, J. Liu, A. Pierce, N. Weiner and I. Yavin, CoGeNT Interpretations, JCAP 08 (2010) 018 [arXiv: 1004.0697] [SPIRES].

[33] H.-C. Cheng, K.T. Matchev and M. Schmaltz, Bosonic supersymmetry? Getting fooled at the CERN LHC, Phys. Rev. D 66 (2002) 056006 [hep-ph/0205314] [SPIRES].

[34] I. Cholis, D.P. Finkbeiner, L. Goodenough and N. Weiner, The PAMELA Positron Excess from Annihilations into a Light Boson, JCAP 12 (2009) 007 [arXiv:0810.5344] [SPIRES].

[35] I. Cholis, G. Dobler, D.P. Finkbeiner, L. Goodenough and N. Weiner, The Case for a 700+ GeV WIMP: Cosmic Ray Spectra from ATIC and PAMELA, Phys. Rev. D 80 (2009) 123518 [arXiv:0811.3641] [SPIRES].

[36] M. Cirelli, M. Kadastik, M. Raidal and A. Strumia, Model-independent implications of the e+, e-, anti-proton cosmic ray spectra on properties of Dark Matter, Nucl. Phys. B 813 (2009) 1 [arXiv: 0809.2409] [SPIRES].

[37] D. Clowe et al., A direct empirical proof of the existence of dark matter, Astrophys. J. 648 (2006) L109 [astro-ph/0608407] [SPIRES].

[38] S. Colafrancesco, S. Profumo and P. Ullio, Detecting dark matter WIMPs in the Draco dwarf: a multi-wavelength perspective, Phys. Rev. D 75 (2007) 023513 [astro-ph/0607073] [SPIRES].

[39] S. Colafrancesco, Dark Matter in Modern Cosmology, AIP Conf. Proc. 1206 (2010) 5 [arXiv: 1004.3869] [SPIRES].

[40] FOR THE MAGIC collaboration, P. Colin et al., Performance of the MAGIC telescopes in stereoscopic mode, arXiv:0907.0960 [SPIRES].

[41] J. Cortina, F. Goebel, T. Schweizer and f.t.M. Collaboration, Technical Performance of the MAGIC Telescopes, arXiv:0907.1211 [SPIRES].

[42] J. Diemand, M. Kuhlen and P. Madau, Dark matter substructure and gamma-ray annihilation in the Milky Way halo, Astrophys. J. 657 (2007) 262 [astro-ph/0611370] [SPIRES].

[43] J. Diemand et al., Clumps and streams in the local dark matter distribution, Nature 454 (2008) 735 [arXiv: 0805.1244] [SPIRES].

[44] MAGIC collaboration, E. Domingo-Santamaria et al., The DISP analysis method for point-like or extended gamma source searches / studies with the MAGIC telescope, in The MAGIC Project. Contributions to ICRC 2005, Pune, India. Part 3: MAGIC detector and analysis details, contributed to 29th International Cosmic Ray Conference (ICRC 2005), Pune India, 3-11 Aug. 2005, pg. 23-27 [astro-ph/0508274] [SPIRES].

[45] R. Essig, N. Sehgal and L.E. Strigari, Bounds on Cross-sections and Lifetimes for Dark Matter Annihilation and Decay into Charged Leptons from Gamma-ray Observations of Dwarf Galaxies, Phys. Rev. D 80 (2009) 023506 [arXiv:0902.4750] [SPIRES]. 
[46] R. Essig, N. Sehgal, L.E. Strigari, M. Geha and J.D. Simon, Indirect Dark Matter Detection Limits from the Ultra-Faint Milky Way Satellite Segue 1, Phys. Rev. D 82 (2010) 123503 [arXiv: 1007.4199] [SPIRES].

[47] D.P. Finkbeiner, L. Goodenough, T.R. Slatyer, M. Vogelsberger and N. Weiner, Consistent Scenarios for Cosmic-Ray Excesses from Sommerfeld-Enhanced Dark Matter Annihilation, JCAP 05 (2011) 002 [arXiv: 1011.3082] [SPIRES].

[48] V.P. Fomin et al., New methods of atmospheric Cherenkov imaging for gamma-ray astronomy. 1: The False source method, Astropart. Phys. 2 (1994) 137.

[49] N. Fornengo, L. Pieri and S. Scopel, Neutralino annihilation into gamma-rays in the Milky Way and in external galaxies, Phys. Rev. D 70 (2004) 103529 [hep-ph/0407342] [SPIRES].

[50] M. Geha, The Least Luminous Galaxy: Spectroscopy of the Milky Way Satellite Segue 1, Astrophys. J. 692 (2009) 1464 [arXiv:0809.2781] [SPIRES].

[51] G. Gilmore et al., The Observed properties of Dark Matter on small spatial scales, Astrophys. J. 663 (2007) 948 [astro-ph/0703308] [SPIRES].

[52] P. Gondolo et al., DarkSUSY: Computing supersymmetric dark matter properties numerically, JCAP 07 (2004) 008 [astro-ph/0406204] [SPIRES].

[53] A.M. Green, S. Hofmann and D.J. Schwarz, The power spectrum of SUSY-CDM on sub-galactic scales, Mon. Not. Roy. Astron. Soc. 353 (2004) L23 [astro-ph/0309621] [SPIRES].

[54] A.M. Hillas, Cherenkov Images of EAS produced by primary gamma rays and by nuclei, Proceedings of the 19th International Cosmic Ray Conference. Vol.3, La Jolla U.S.A. (1985), pg. 445.

[55] D. Hooper, P. Blasi and P.D. Serpico, Pulsars as the Sources of High Energy Cosmic Ray Positrons, JCAP 01 (2009) 025 [arXiv:0810.1527] [SPIRES].

[56] T.C. Consortium, Design Concepts for the Cherenkov Telescope Array, arXiv:1008.3703 [SPIRES].

[57] G. Jungman, M. Kamionkowski and K. Griest, Supersymmetric dark matter, Phys. Rept. 267 (1996) 195 [hep-ph/9506380] [SPIRES].

[58] A.A. Klypin, A.V. Kravtsov, O. Valenzuela and F. Prada, Where are the missing galactic satellites?, Astrophys. J. 522 (1999) 82 [astro-ph/9901240] [SPIRES].

[59] WMAP collaboration, E. Komatsu et al., Seven-Year Wilkinson Microwave Anisotropy Probe (WMAP) Observations: Cosmological Interpretation, Astrophys. J. Suppl. 192 (2011) 18 [arXiv: 1001.4538] [SPIRES].

[60] A.V. Kravtsov, A.A. Klypin, J.S. Bullock and J.R. Primack, The Cores of Dark Matter Dominated Galaxies: theory vs. observations, Astrophys. J. 502 (1998) 48 [astro-ph/9708176] [SPIRES].

[61] A.V. Kravtsov, O.Y. Gnedin and A.A. Klypin, The tumultuous lives of galactic dwarfs and the missing satellites problem, Astrophys. J. 609 (2004) 482 [astro-ph/0401088] [SPIRES].

[62] M. Lattanzi and J.I. Silk, Can the WIMP annihilation boost factor be boosted by the Sommerfeld enhancement?, Phys. Rev. D 79 (2009) 083523 [arXiv:0812.0360] [SPIRES].

[63] ZEPLIN-III collaboration, V.N. Lebedenko et al., Limits on the spin-dependent WIMP-nucleon cross-sections from the first science run of the ZEPLIN-III experiment, Phys. Rev. Lett. 103 (2009) 151302 [arXiv:0901.4348] [SPIRES].

[64] T.-P. Li and Y.-Q. Ma, Analysis methods for results in gamma-ray astronomy, Astrophys. J. 272 (1983) 317.

[65] S.P. Martin, A Supersymmetry Primer, hep-ph/9709356 [SPIRES]. 
[66] N.F. Martin, R.A. Ibata, S.C. Chapman, M. Irwin and G.F. Lewis, A Keck/DEIMOS spectroscopic survey of faint Galactic satellites: Searching for the least massive dwarf galaxies, Mon. Not. Roy. Astron. Soc. 380 (2007) 281 [arXiv:0705.4622] [SPIRES].

[67] G.D. Martinez, J.S. Bullock, M. Kaplinghat, L.E. Strigari and R. Trotta, Indirect Dark Matter Detection from Dwarf Satellites: Joint Expectations from Astrophysics and Supersymmetry, JCAP 06 (2009) 014 [arXiv:0902.4715] [SPIRES].

[68] G.D. Martinez et al., A Complete Spectroscopic Survey of the Milky Way satellite Segue 1: Dark matter content, stellar membership and binary properties from a Bayesian analysis, arXiv: 1008.4585 [SPIRES].

[69] P. Meade, M. Papucci, A. Strumia and T. Volansky, Dark Matter Interpretations of the Electron/Positron Excesses after FERMI, Nucl. Phys. B 831 (2010) 178 [arXiv:0905.0480] [SPIRES].

[70] N. Mirabal, Swift observation of Segue 1: constraints on sterile neutrino parameters in the darkest galaxy, arXiv:1010.4706 [SPIRES].

[71] B. Moore et al., Dark Matter Substructure in Galactic Halos, astro-ph/9907411 [SPIRES].

[72] B. Moore, T.R. Quinn, F. Governato, J. Stadel and G. Lake, Cold collapse and the core catastrophe, Mon. Not. Roy. Astron. Soc. 310 (1999) 1147 [astro-ph/9903164] [SPIRES].

[73] J.F. Navarro, C.S. Frenk and S.D.M. White, A Universal Density Profile from Hierarchical Clustering, Astrophys. J. 490 (1997) 493 [astro-ph/9611107] [SPIRES].

[74] J.F. Navarro et al., The Diversity and Similarity of Cold Dark Matter Halos, arXiv:0810.1522 [SPIRES].

[75] H.P. Nills, Supersymmetry, Supergravity and Particle Physics, Phys. Rept. 110 (1984) 1.

[76] M. Niederste-Ostholt et al., The Origin of Segue 1, Mon. Not. Roy. Astron. Soc. 398 (2009) 1771 [arXiv:0906.3669] [SPIRES].

[77] M. Persic, P. Salucci and F. Stel, The Universal rotation curve of spiral galaxies: 1. The Dark matter connection, Mon. Not. Roy. Astron. Soc. 281 (1996) 27 [astro-ph/9506004] [SPIRES].

[78] L. Pieri, G. Bertone and E. Branchini, Dark Matter Annihilation in Substructures Revised, Mon. Not. Roy. Astron. Soc. 3841627 [arXiv:0706.2101] [SPIRES].

[79] L. Pieri, M. Lattanzi and J. Silk, Constraining the Sommerfeld enhancement with Cherenkov telescope observations of dwarf galaxies, Mon. Not. Roy. Astron. Soc. 399 (2009) 2033 [arXiv:0902.4330] [SPIRES].

[80] M. Pospelov and A. Ritz, Astrophysical Signatures of Secluded Dark Matter, Phys. Lett. B 671 (2009) 391 [arXiv: 0810.1502] [SPIRES].

[81] S. Profumo, Dissecting Pamela (and ATIC) with Occam's Razor: existing, well-known Pulsars naturally account for the 'anomalous' Cosmic-Ray Electron and Positron Data, arXiv: 0812.4457 [SPIRES].

[82] W.A. Rolke, A.M. Lopez and J. Conrad, Limits and confidence intervals in the presence of nuisance parameters, Nucl. Instrum. Meth. A 551 (2005) 493 [physics/0403059].

[83] P. Salucci, F. Nesti, G. Gentile and C.F. Martins, The dark matter density at the Sun's location, Astron. Astrophys. 523 (2010) A83 [arXiv:1003.3101] [SPIRES].

[84] M.A. Sánchez-Conde et al., Dark matter annihilation in Draco: New considerations of the expected gamma flux, Phys. Rev. D 76 (2007) 123509 [astro-ph/0701426] [SPIRES].

[85] M.A. Sánchez-Conde, M. Cannoni, F. Zandanel, M.E. Gomez and F. Prada, Dark matter searches with Cherenkov telescopes: nearby dwarf galaxies or local galaxy clusters?, arXiv:1104.3530 [SPIRES]. 
[86] P. Scott et al., Direct Constraints on Minimal Supersymmetry from Fermi-LAT Observations of the Dwarf Galaxy Segue 1, JCAP 01 (2010) 031 [arXiv:0909.3300] [SPIRES].

[87] G. Servant and T.M.P. Tait, Is the lightest Kaluza-Klein particle a viable dark matter candidate?, Nucl. Phys. B 650 (2003) 391 [hep-ph/0206071] [SPIRES].

[88] J.D. Simon et al., A Complete Spectroscopic Survey of the Milky Way Satellite Segue 1: The Darkest Galaxy, Astrophys. J. 733 (2011) 46 [arXiv:1007.4198] [SPIRES].

[89] J.D. Simon and M. Geha, The Kinematics of the Ultra-Faint Milky Way Satellites: Solving the Missing Satellite Problem, Astrophys. J. 670 (2007) 313 [arXiv:0706.0516] [SPIRES].

[90] V. Springel, Prospects for detecting supersymmetric dark matter in the Galactic halo, Nature 456 (2008) 73.

[91] V. Springel et al., The Aquarius Project: the subhalos of galactic halos, Mon. Not. Roy. Astron. Soc. 391 (2008) 1685 [arXiv:0809.0898] [SPIRES].

[92] L.E. Strigari, S.M. Koushiappas, J.S. Bullock and M. Kaplinghat, Precise constraints on the dark matter content of Milky Way dwarf galaxies for gamma-ray experiments, Phys. Rev. D 75 (2007) 083526 [astro-ph/0611925] [SPIRES].

[93] L.E. Strigari et al., Redefining the Missing Satellites Problem, Astrophys. J. 669 (2007) 676 [arXiv:0704.1817] [SPIRES].

[94] P. Ullio, L. Bergstrom, J. Edsjo and C.G. Lacey, Cosmological dark matter annihilations into gamma-rays: A closer look, Phys. Rev. D 66 (2002) 123502 [astro-ph/0207125] [SPIRES].

[95] R.G. Wagner and f.t.V. Collaboration, Indirect Dark Matter Searches with VERITAS, arXiv:0910.4563 [SPIRES].

[96] M. Wood et al., A Search for Dark Matter Annihilation with the Whipple 10m Telescope, arXiv:0801.1708 [SPIRES].

[97] SDSS-II SEGUE collaboration, B. Yanny et al., SEGUE: A Spectroscopic Survey of 240,000 stars with $g=14-20$, Astron. J. 137 (2009) 4377 [arXiv:0902.1781] [SPIRES].

[98] H. Yuksel, M.D. Kistler and T. Stanev, TeV Gamma Rays from Geminga and the Origin of the GeV Positron Excess, Phys. Rev. Lett. 103 (2009) 051101 [arXiv:0810.2784] [SPIRES].

[99] M. Xiang-Gruess, Y.-Q. Lou and W.J. Duschl, Dark matter dominated dwarf disc galaxy Segue 1, arXiv:0909.3496 [SPIRES].

[100] G. Bertone eds., Particle Dark Matter, first edition, Cambridge University Press, Cambridge U.K. (2010).

[101] C. Farnier, Dark Matter Annihilations Search In Dwarfs Spheroidal Galaxies With Fermi, talk at Roma International Conference in Astro-Particle Physics, Roma Italy (2009).

[102] T. Jeltema, Searching for dark matter annihilation in dwarf spheroidal galaxies with Fermi, tolk at TEV Particle Astrophysics Conference, Menlo Park U.S.A. 13-17 July 2009.

[103] S. Murgia, Search for dark matter and new physics, tolk at TEV Particle Astrophysics Conference, Menlo Park U.S.A. 13-17 July 2009. 